\title{
Fluids of semiflexible ring polymers: effective potentials and clustering
}

\author{
Marco Bernabei, ${ }^{1}$ Petra Bacova, ${ }^{2}$ Angel J. \\ Moreno, ${ }^{3,1, *}$ Arturo Narros, ${ }^{4}$ and Christos N. Likos ${ }^{4}$ \\ ${ }^{1}$ Donostia International Physics Center, \\ Paseo Manuel de Lardizabal 4, 20018 San Sebastián, Spain. \\ ${ }^{2}$ Departamento de Física de Materiales, \\ Universidad del País Vasco (UPV/EHU), \\ Apartado 1072, 20080 San Sebastián, Spain. \\ ${ }^{3}$ Centro de Física de Materiales (CSIC, \\ UPV/EHU) and Materials Physics Center MPC, \\ Paseo Manuel de Lardizabal 5, E-20018 San Sebastián, Spain. \\ ${ }^{4}$ Faculty of Physics, University of Vienna, \\ Boltzmanngasse 5, A-1090 Vienna, Austria.
}

(Dated: September 24, 2012)

We present a computational investigation on the structural properties of a fluid of semiflexible ring polymers. Stiffness is introduced by implementing intramolecular barriers. Because of these barriers, shrinkage of the rings is energetically unfavourable, and the ring size can exhibit a non-monotonic density dependence. At high concentrations the rings can swell and adopt open configurations that facilitate interpenetration and clustering. We obtain effective potentials between the centersof-mass of the rings at infinite dilution, and explore their validity over the whole range of concentrations. Except for the limit of small rings, the effective fluid of ultrasoft particles provides a good description of the real system over a considerable range of densities, even above the overlap concentration. In particular the clustering behaviour predicted by the effective description is observed in the real system for a certain range of molecular masses. However, the effective description is incomplete. Inspection of the clusters of real rings reveals that these can arrange in a complex disordered phase formed by long columns of oblate rings, which are penetrated by 
bundles of elongated prolate rings. These anisotropic features of the real system are not captured by the standard effective approach, which only considers macromolecular centers-of-mass. This suggests the need to include the relative orientation between rings in the effective potentials.

${ }^{*}$ Corresponding author: wabmosea@ehu.es 


\section{INTRODUCTION}

Ring polymers (RP's) are formed by the simple operation of joining together the free ends of a polymer chain [1]. They occur either naturally — the most prominent example being circular DNA - or they can be synthesized both by chemical and by biochemical procedures. RP's have attracted the interest of a broad range of researchers across various disciplines, including mathematics, biology and biophysics, chemistry, and physics. A fascinating aspect of RP's is that, albeit they result from a simple operation that affects neither the physics nor the chemistry of the polymer, there is a fundamental change in their topology: once a ring is formed, it cannot open up again. Accordingly, any knot that might be present in the linear chain (and which can be released by topology-preserving operations while the chain is open), will become permanent once the ring is closed. Any subsequent, nondestructive manipulation or fluctuation of the molecule will not alter its topological state. The simplest $\mathrm{RP}$ is the unknot (also called trivial knot).

Topological properties manifest themselves on a variety of properties of RP's. Much work has been dedicated to their scaling behaviour at infinite dilution [2-11]. It has been demonstrated that the size of isolated RP's, quantified by their diameter of gyration $D_{g}$, scales as $\left\langle D_{g}^{2}\right\rangle \sim N^{2 \nu}$, with $\nu \cong 0.588$ the Flory exponent of self-avoiding linear chains. This does not only occur for self-avoiding rings but, strikingly, even for ideal circles. Another spectacular expression of the topological constraints is the fact that two ideal ring polymers experience a repulsive effective interaction between their centers of mass, whilst the same vanishes between two ideal chains or an ideal chain and a ring [12]. This so-called topological potential is a quantity unique to ring polymers $[13,14]$, and it is present for any kind of pairs of rings, independently of their own knotedness and flexibility.

Studies on many-body systems of RP's have been scarce in comparison with singlemolecule investigations. Recent large-scale simulations of extremely long, non-concatenated unknotted rings at melt densities $[15,16]$ have revealed a complex crossover from $\left\langle D_{g}^{2}\right\rangle \sim$ $N^{4 / 5}$ to $\left\langle D_{g}^{2}\right\rangle \sim N^{2 / 3}$ by increasing $N$, instead of the random-walk scaling $\left\langle D_{g}^{2}\right\rangle \sim N$ generally found in melts of linear chains. The analysis of [16] rationalizes the former observation by showing that long rings in melts adopt complex conformations, consisting of crumpled globules with long protrusions. Concerning dynamic properties, since the pioneering work of Edwards $[17,18]$ it has been recognized that the topological constraints in rings lead to 
novel types of entanglement effects. Investigations on melts of unknotted, non-concatenated rings [19-23] have shown that they display a higher diffusivity [19-25] and that the Rouse regime extends to larger scales than in their linear counterparts [26]. Rheological experiments [27] and simulations [25] have revealed a power-law stress relaxation, instead of the usual reptation-like exponential behaviour found for linear chains.

Relatively little attention has been paid to the broad regime that bridges the gap between dilute solutions and melts, covering a density range from below the overlap concentration all the way deeply into the semiconcentrate regime. This can be facilitated by coarse-graining, a fruitful strategy that has been successfully applied to many classes of polymeric systems. In this methodology the internal degrees of freedom of the polymer (i.e, the monomer coordinates) are replaced by a single one, typically chosen as the position of the center-of-mass. The so-obtained effective interaction $V_{\text {eff }}(R)$ between two polymers is ultrasoft, since polymers are strongly penetrable objects. Moreover, their centers-of-mass can often coincide without violating excluded-volume interactions. In this situation the ultrasoft potential is bounded, i.e., it does not diverge at any separation between the centers-of-mass. The investigation of the corresponding fluid of ultrasoft particles provides an efficient and economical route to solve the structural and thermodynamic properties of the real system [28, 29]. Linear polymer chains [30, 31], neutral $[32,33]$ and charged star polymers [34, 35], dendrimers [36-38], microgels [39, 40] and block copolymers [41-43], constitute recent examples for which coarse-graining has led to a thorough understanding of broad aspects of equilibrium properties and dynamical arrest, with many open questions still waiting to be answered in this very active field of research.

By means of extensive computer simulations, Narros et al. [44] derived coarse-grained potentials for flexible ring polymers in athermal solvents, and explored their range of validity to predict structural properties in the semiconcentrate regime. They showed that the effective potential carries the signature of the topology, and that it changes dramatically with increasing knot complexity, at least for moderate molecular weights. The works on ring polymers by Hirayama et al. [13] and by Bohn and Heermann [14] employed different microscopic models for the monomer interactions, but reported results of $V_{\text {eff }}(R)$ that were analogous to those of Ref. [44]. This confirms the robustness of the topological effects on the effective potentials.

One of the key findings of Ref. [44] was that the practical usefulness of the effective 
potential between flexible RP's is limited to rather dilute solutions. A striking consequence of the features of the obtained effective potential is a strong clustering behaviour of the ultrasoft particles with increasing concentration. However clustering was not found in the real system. As the overlap concentration is approached, the deformation of the rings alters their shape through shrinking, generating thereby many-body interactions that cannot be accounted for by the infinite-dilution effective potential. Indeed shrinking of the rings strongly hinders interpenetration, and prevents the formation of clusters that was observed for the ultrasoft particles [44].

A natural way of preventing a strong shrinking of the rings with increasing concentration is to introduce intramolecular stiffness. This can be done by implementing intramolecular barriers, which indeed are ubiquitous in real polymers but were not included in the model of Ref. [44]. If such barriers are strong enough, deformation of the rings is energetically very unfavourable. In such conditions, one might expect that in concentrate solutions the rings will interpenetrate and eventually form clusters in order to fill the space. With these ideas in mind, in this article we present an extensive computational investigation of the structural properties of a fluid of semiflexible rings in the whole range of concentration. For the small investigated molecular weights, we find a weak shrinking of the rings with increasing concentration, and even swelling far beyond the overlap density. We derive effective potentials at infinite dilution and test their validity at high concentrations, by comparing simulations of the effective fluid of ultrasoft particles and the real system of semiflexible rings. The radial distribution functions of the real system provide evidence for clustering, which is rather pronounced for some of the simulated cases. The effective fluid is able to account for this feature, at worst to a qualitative level. However, a detailed analysis of intramolecular conformations and orientational correlations in the real system reveals that the approach of the effective fluid is incomplete. Namely, it misses the anisotropic features found in the real system. These include the formation of complex disordered phases consisting of long columns of oblate rings, which are penetrated by bundles of elongated prolate rings. This suggests the need to improve the standard approach, which only considers macromolecular centers-of-mass, by including the relative orientation between rings in the coordinates of the effective potential.

The article is organized as follows. In Section II we give details of the investigated model and of the used simulation techniques. In Section III we characterize the density- 
dependence of the ring size, derive effective potentials and establish the limits of validity of such potentials. In Section IV we discuss the physical origin of the former results by analyzing the shape of the rings as well as orientational correlations, and clarify the nature of the observed clustering behaviour. Conclusions are given in Section V.

\section{MODEL AND SIMULATION DETAILS}

\section{A. Model}

We have simulated unknotted, non-concatenated rings of $N$ monomers by using the wellknown bead-spring model of Kremer and Grest [45]. The non-bonded interaction between any two monomers ('beads') is given by a shifted and cut-off Lennard-Jones potential:

$$
V_{\mathrm{LJ}}(r)=4 \epsilon\left[\left(\frac{\sigma}{r}\right)^{12}-\left(\frac{\sigma}{r}\right)^{6}+\frac{1}{4}\right] H\left(r_{\mathrm{c}}-\sigma\right),
$$

where $r_{\mathrm{c}}=2^{1 / 6} \sigma$ and $H(x)$ is the Heaviside function. Potential and forces are continuous at $r_{\mathrm{c}}$. If the two monomers are bonded to each other they also interact through a finitely extensible non-linear elastic potential (FENE) [45],

$$
V_{\mathrm{FENE}}(r)=-\epsilon K_{\mathrm{F}} R_{0}^{2} \ln \left[1-\left(\frac{r}{R_{0} \sigma}\right)^{2}\right],
$$

with $K_{\mathrm{F}}=15$ and $R_{0}=1.5$. The total potential $V_{\mathrm{LJ}}+V_{\mathrm{FENE}}$ between connected monomers has a sharp minimum at $r=0.96 \sigma$. This, together with the steepness of $V_{\mathrm{LJ}}$ and $V_{\mathrm{FENE}}$, keeps the bond distance within small fluctuations. Thus, chain uncrossability is fulfilled, preventing knotting or concatenation of the rings.

Intramolecular stiffness was introduced by means of a bending potential given by

$$
V_{\text {bend }}(\phi)=\epsilon K_{\text {bend }}(1-\cos \phi)^{2}
$$

where $\phi$ is the angle between two consecutive bonds. We used a bending constant $K_{\text {bend }}=$ 30. For simplicity we used the same mass $m$ for all the monomers, and reduced units $m=\sigma=\epsilon=k_{\mathrm{B}}=1$ ( $k_{\mathrm{B}}$ is the Boltzmann constant). All the simulations were performed at temperature $T=1$. In the next subsections we give details about the different simulation methods used in this work. 


\section{B. Monte Carlo simulations}

We have obtained effective potentials for semiflexible rings of $N=20,30,50$ and 100 monomers. If we use the distance between the macromolecular centers-of-mass $R$ as the effective coordinate, the effective potential $V_{\text {eff }}(R)$ between two isolated macromolecules is given by [28]

$$
\frac{V_{\text {eff }}(R)}{k_{\mathrm{B}} T}=-\ln [g(R)]
$$

where $g(R)$ is the radial distribution function of the macromolecular centers-of-mass. Obviously there is no possible contact between the two macromolecules at distances $R \gg D_{\mathrm{g}}$, with $D_{\mathrm{g}}$ the average diameter of gyration. Therefore $V_{\mathrm{eff}}\left(R \gg D_{\mathrm{g}}\right)=0$. This indeed reflects, through Eq. (4), the limit $g\left(R \gg D_{\mathrm{g}}\right)=1$. On the contrary the contributions from excluded volume interactions between monomers, as well as from topology (non-concatenation), to the effective potential will be important when the two macromolecules are interpenetrated, i.e., for small separation $R$. This can lead to a significant correlation hole in $g(R)$, and through Eq. (4), to potential amplitudes of several times $k_{\mathrm{B}} T$.

In order to derive the effective pair potential between isolated rings through Eq. (4) we need to obtain the distribution $g(R)$ by, e.g., Monte Carlo (MC) simulations. However, standard MC simulations are highly inefficient to sample $g(R)$ at short distances. The reason is that spontaneous interpenetration of the macromolecules is strongly hindered by the increasing number of excluded volume interactions between monomers at small $R$. To overcome this difficulty, we used the umbrella sampling technique [46, 47]. Thus, we performed MC simulations in several windows $R_{\text {min }} \leq R \leq R_{\max }$, where MC trials producing separations $R<R_{\min }$ or $R>R_{\max }$ were rejected. It can be seen that this method provides the correct value of $\ln [g(R)]$ plus a window-dependent constant. Thus, a continuous curve can be constructed by applying different vertical shifts to the results obtained in the different sampling windows, and this continuous curve will be the total $\ln [g(R)]$ plus a constant. By using the condition $V_{\text {eff }}\left(R \gg D_{\mathrm{g}}\right)=0$, the latter constant can be removed and finally we obtain the potential $V_{\text {eff }}(R)$ through Eq. (4). To facilitate the construction of the continuous curve of $\ln [g(R)]$, we used overlapping intervals for consecutive windows $k$ and $k+1$, i.e,

$R_{\min }^{(k)}<R_{\min }^{(k+1)}<R_{\max }^{(k)}<R_{\max }^{(k+1)}$. The different used windows explored the whole range of relevant distances from $R=0$ up to a maximum $R \sim 3 D_{\mathrm{g}}$, which guaranteed probing the large- $R$ limit of zero effective interaction. 
In addition to the standard MC monomer displacements, we implemented large-scale motions in order to explore more efficiently the configuration space and to speed up the simulations. These motions included rigid translations of the rings and rigid rotations of intramolecular segments between randomly selected monomers (crankshaft motions [4850]). The maximum allowed amplitude for these rotations was controlled for each selected segment, in order to prevent accidental knotting or concatenation. Each MC step consisted of a loop of single monomer displacements over all the monomers of the two rings, plus a few large-scale motions as those mentioned above. All MC motions were accepted or rejected according to the standard Metropolis criterion [46] and the additional condition of keeping the separation $R$ within the corresponding sampling window. The number of MC steps of the production runs was typically $10^{8}<n_{\mathrm{s}}<10^{9}$ for each sampling window. For improving statistics, average was also performed over several initial configurations.

In order to place the rings in the different sampling windows we proceeded as follows. The two rings were generated by inserting the beads in two circles. The initial separation between the rings was much longer than the diameter of the circles, preventing concatenation. Then an MC run with fixed centers-of-mass was performed to equilibrate the intramolecular conformations. After this, a new MC run was performed in which MC steps were accepted with the standard Metropolis criterion and with the additional condition of decreasing the distance $R$ between centers-of-mass. MC steps leading to an increase in $R$ were rejected. When the distance reached one of the selected sampling windows $R_{\min } \leq R \leq R_{\max }$ (see above) the run was stopped, and the configuration of the two rings was taken as the initial one for the umbrella sampling MC run in that window. This procedure was repeated for the rest of the sampling windows.

\section{Molecular dynamics simulations}

We also performed large-scale molecular dynamics (MD) simulations of fluids of rings at different concentrations. Two cases were investigated: i) the fluid of real rings, with the monomers interacting through the potentials of Eqs. (1-3), and ii) the effective fluid of ultrasoft particles, interacting through the effective potential $V_{\text {eff }}(R)$ obtained by the umbrella sampling MC (see above). As in the MC simulations, no explicit solvent was included. The simulations were performed under Langevin dynamics. The corresponding 
equations of motion are [51]:

$$
m_{i} \ddot{\mathbf{r}}_{i}(t)=\mathbf{F}_{i}(t)-\gamma m_{i} \dot{\mathbf{r}}_{i}(t)+\mathcal{R}_{i}(t),
$$

where $\mathbf{r}_{i}(t)$ denotes the position, at time $t$, of the particle $i$, this being a ring monomer or an ultrasoft particle in the aforementioned cases i) and ii) respectively. Likewise, $m_{i}$ is the monomer mass $m$ or the ring mass $N m$. The force $\mathbf{F}_{i}$ is the Newtonian force acting on the particle $i$, and it is defined as $-\nabla_{i}\left(V_{\mathrm{LJ}}+V_{\mathrm{FENE}}+V_{\mathrm{bend}}\right)$ for the monomer rings and as $-\nabla_{i} V_{\text {eff }}$ for the ultrasoft particles. The quantities $\mathcal{R}_{i}(t)$ are random forces following the relations $\left\langle\mathcal{R}_{i}^{\alpha}(t)\right\rangle=0$ and $\left\langle\mathcal{R}_{i}^{\alpha}(t) \mathcal{R}_{j}^{\beta}\left(t^{\prime}\right)\right\rangle=2 \gamma m_{i} k_{\mathrm{B}} T \delta_{i j} \delta_{\alpha \beta} \delta\left(t-t^{\prime}\right)$, where greek indexes denote cartesian components. We used a friction $\gamma=0.5$, which provides correct thermalization at the selected temperature $T=1$. The Langevin equations of motion were discretized with a time step $\Delta t=0.01$ and integrated in the velocity-Verlet scheme, following the impulse approach proposed in Refs. [52, 53].

The MD simulations of the real rings were performed with the free package ESPResSo $[54,55]$. We simulated rings of $N=20,50$ and 100 monomers. The simulation boxes contained $n=2400,1600$ and 1200 rings respectively. Periodic boundary conditions were applied to the cubic simulation box and a linked-cell method [46] was implemented to reduce computational expense in the calculation of interparticle distances. To generate the most dilute of the investigated solutions of real rings, we placed circular rings in the nodes of a square lattice with spacing much larger than the circle diameter. With this, we prevented initial ring concatenation. Then an equilibration run was performed, at constant volume, to both relax the intramolecular conformations and to let the rings diffuse long distances. To generate the ring solution at the other investigated concentrations, a slow compression was applied to the system equilibrated at the inmediately lower concentration. This was done by periodically rescaling coordinates and box sides up to the desired density. We used a scaling factor slightly smaller than one $(f=0.99)$, and a period of several thousand steps to allow for local reequilibration after each rescaling step. With these choices numerical unstabilities and accidental concatenation was prevented. After reaching the desired density an equilibration run at constant volume was performed. In all cases the equilibration runs were long enough (up to $10^{7}$ steps) to let the rings diffuse several times their own diameters.

In the case of the ultrasoft particles, interpenetration does not yield numerical unstabilities since the interaction potential $V_{\text {eff }}(R)$ is bounded for all distances (see below). Thus, 
the systems at the different concentrations were just generated by random insertion of the particles in the simulation box. For each density and molecular weight, the number of ultrasoft particles in the simulation box was the same as the number of rings in the respective simulation of the real system (see above). As in the case of the real rings, equilibration runs were performed at constant volume, letting the ultrasoft particles diffuse several times their own diameters. Finally, production runs of up to $2 \times 10^{7} \mathrm{MD}$ steps were performed at constant volume for both systems of real rings and ultrasoft particles, in order to generate an ensemble of equilibrium configurations. These were saved every $10^{4} \mathrm{MD}$ steps and used to compute the physical quantities discussed in next sections.

\section{RESULTS}

Fig. 1 shows the molecular-weight dependence of the mean squared diameter of gyration $\left\langle D_{\mathrm{g} 0}^{2}\right\rangle$ of isolated rings (i.e, at infinite dilution). This was calculated from the MC runs in the sampling windows with longest $R$, for which there was no possible contact between the two rings. The data in Fig. 1 include the results for the semiflexible rings of this work and, for comparison, those of the flexible rings of Ref. [44]. As expected, the introduction of stiffness by the intramolecular barriers leads to an increase in the ring size. Stiffness affects the scaling behaviour up to intermediate scales $(N \lesssim 100)$, leading to a stronger $N$-dependence than in flexible rings. On the contrary, for molecular weights $N \gtrsim 100$ semiflexible and flexible rings exhibit, within statistics, the same scaling behaviour $\left\langle D_{\mathrm{g} 0}^{2}\right\rangle \sim N^{2 \nu}$, where $\nu \cong 0.588$ is the Flory exponent [56]. The two data sets in Fig. 1 are related, in the large- $N$ limit, by a factor $\kappa \approx 3.7$. This is the ratio between their effective squared step lengths, and can be used as an estimation of the ratio of the characteristic ratios $C_{\infty}$ of long semiflexible and flexible rings in melt conditions [56]. Flexible bead-spring polymers at melt densities show typical values $C_{\infty}^{\text {flex }} \sim 1.6$. Therefore an estimation of the characteristic ratio at melt density for the semiflexible model used by us is $\kappa C_{\infty}^{f \text { flex }} \sim 6$. This is a typical value for common polymers [57], supporting the simulated model as a qualitatively realistic one. 


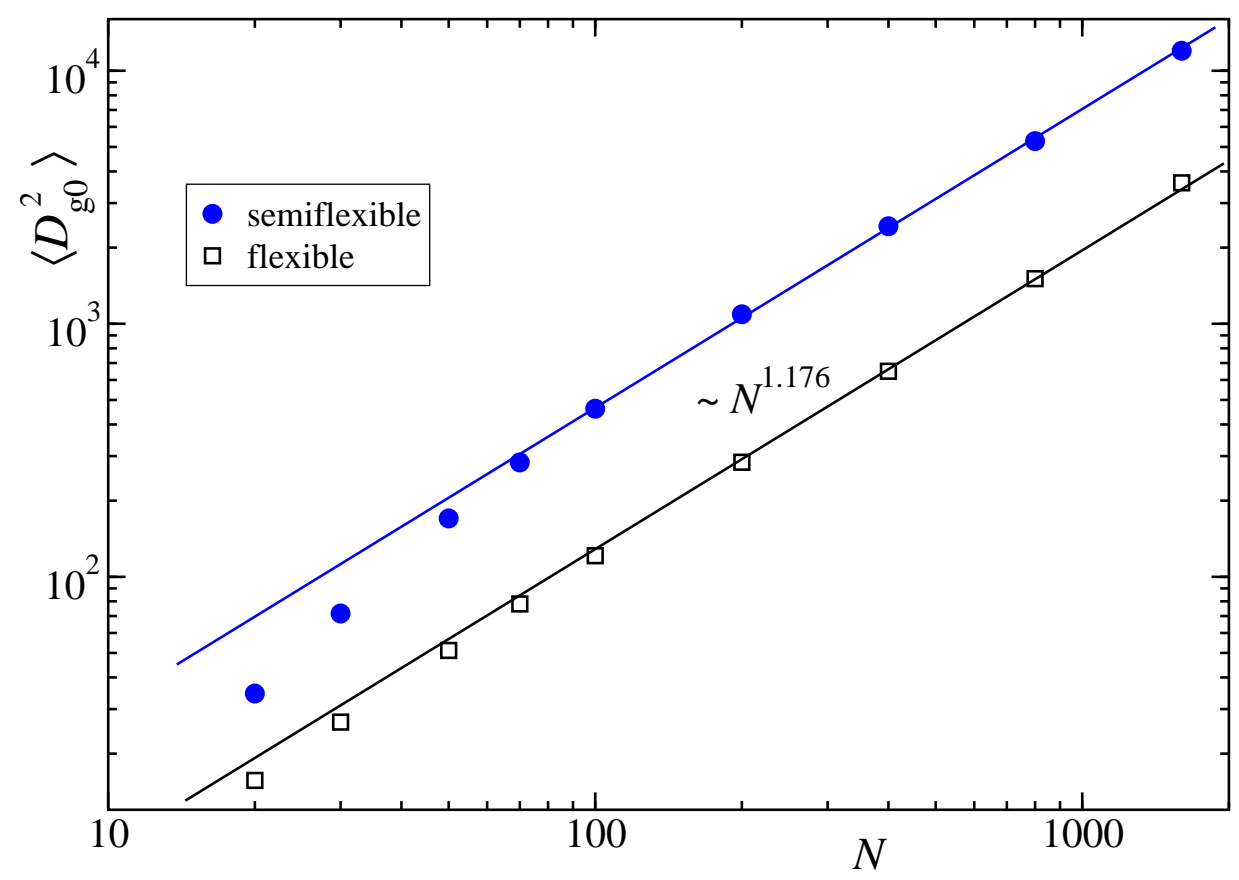

FIG. 1: Molecular weight dependence of the squared diameter of gyration at infinite dilution. Filled and empty symbols correspond to semiflexible and flexible rings respectively. The lines indicate scaling behaviour $\left\langle D_{\mathrm{g} 0}^{2}\right\rangle \sim N^{2 \nu}$, where $\nu \cong 0.588$ is the Flory exponent.

\section{A. Ring size}

The effective density, i.e. the density of the effective fluid obtained by considering each macromolecule as a single ultrasoft particle, can be defined by rescaling distances by some length scale characterizing the macromolecular size. A natural length scale is the average diameter of gyration at infinite dilution. Thus, we have used the definition $\rho_{\text {eff }}=n\left(L / D_{\mathrm{g} 0}\right)^{-3}$, where $n$ is the number of rings in the simulation box of side $L$. The case $\rho_{\text {eff }}=1$ is defined as the overlap density. Around this density the packing of the effective fluid is similar to those of atomic liquids. Fig. 2 displays the effective densities of the simulated systems of semiflexible rings versus the respective monomer densities (obtained as $\rho=n N L^{-3}$ ). For comparison we also include the corresponding results for the flexible rings investigated in Ref. [44]. The explored range of densities covers 2-3 decades. For the case of flexible rings the whole relevant range of monomer concentrations was studied, from high dilution $(\rho \rightarrow 0)$ to melt densities ( $\rho \rightarrow 1$ in bead-spring models as those used here). Melt densities could not be explored in the case of semiflexible rings. The introduction of strong intramolec- 


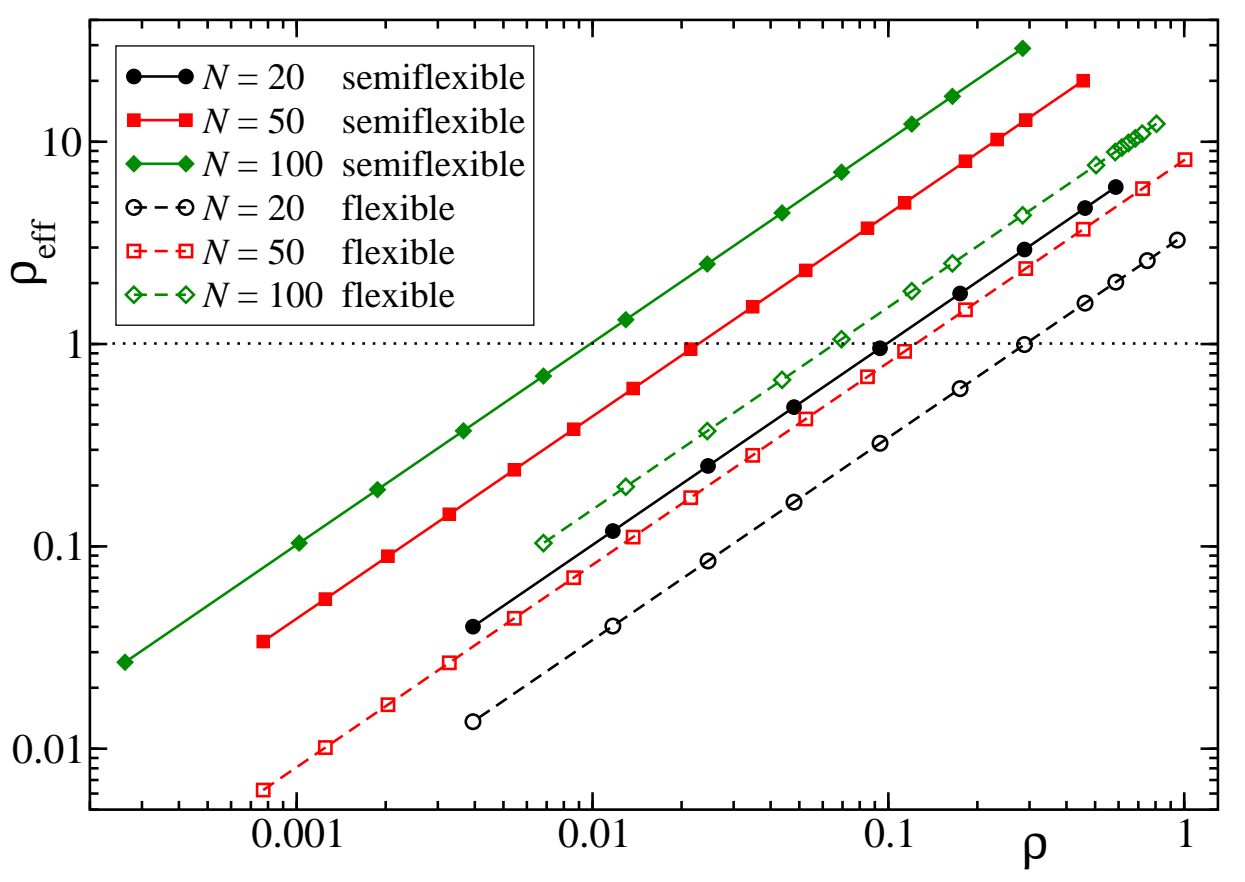

FIG. 2: Relation between the effective density and the monomer density for the investigated molecular weights (see legend). Filled symbols are data of this work for semiflexible rings. Empty symbols are data for the flexible rings investigated in Ref. [44]. Note that a different definition of $\rho_{\text {eff }}$ was used in [44], and the numerical values in this figure have been modified accordingly. Solid and dashed lines are guides for the eyes. The horizontal dotted line indicates the overlap density of the efffective fluid, $\rho_{\text {eff }}=1$.

ular barriers increases dramatically the relaxation times at high densities [58-60], and for the large systems of semiflexible rings simulated here equilibration times at melt densities are computationally prohibitive. Thus, the highest monomer densities investigated for the semiflexible rings ranged from $\rho \approx 0.3$ (for $N=100$ ) to $\rho \approx 0.6$ (for $N=20$ ).

For fixed molecular weight and monomer concentration, the density of the effective fluid is higher for the semiflexible rings than for the flexible ones. Of course, this is a direct consequence of the larger volume spanned by a semiflexible ring, which has a larger diameter of gyration than its flexible counterpart (Fig. 1). As can bee seen in Fig. 2, the studied effective densities range from values far below the overlap density $\left(\rho_{\text {eff }} \ll 0.1\right)$ to values far above it (of up to $\rho_{\text {eff }} \approx 30$ for semiflexible rings of $N=100$ ). In other words, the simulations cover a density range from highly dilute solutions to fluids of strongly interpenetrated ultrasoft 


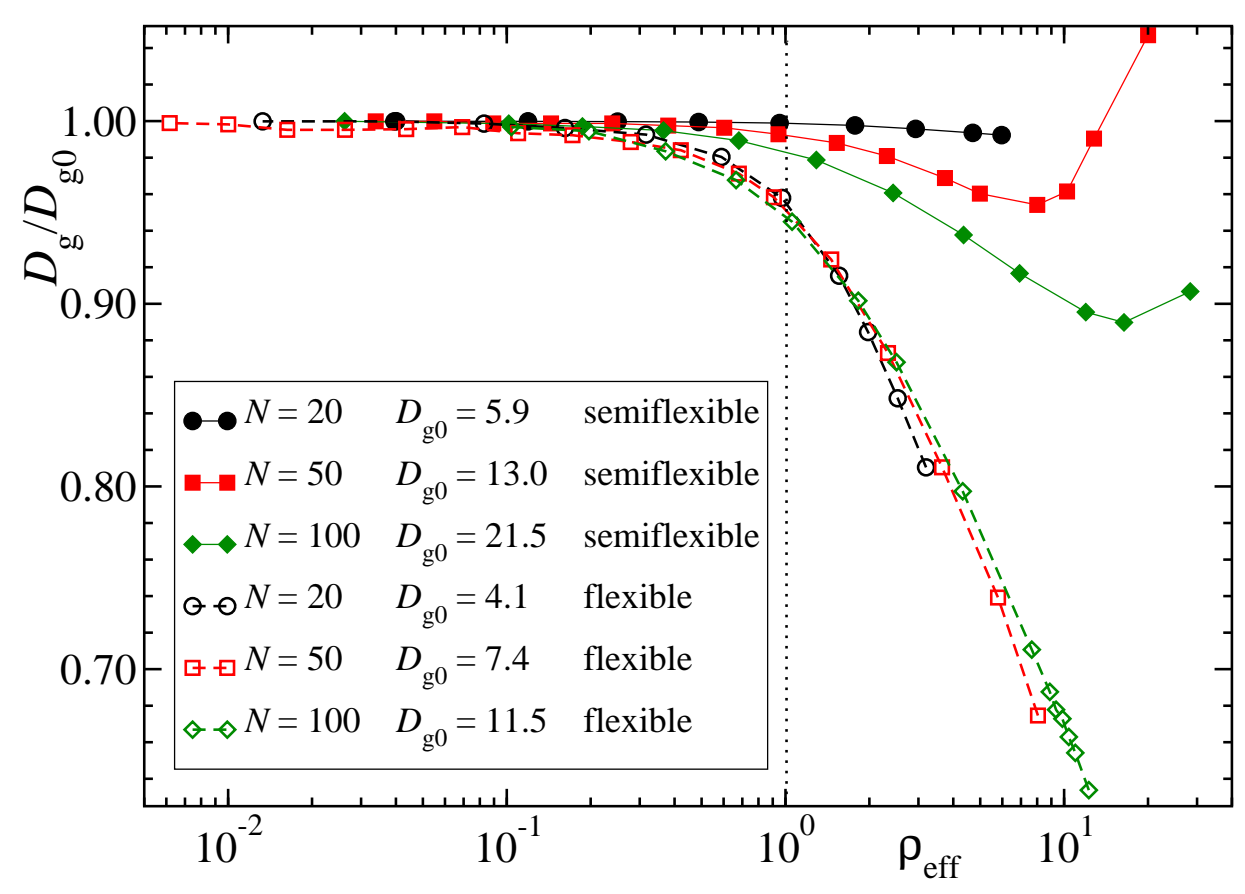

FIG. 3: Diameter of gyration $D_{\mathrm{g}}$, normalized by its value at infinite dilution $D_{\mathrm{g} 0}$ (see legend), as a function of the effective density. Different data sets correspond to different molecular weights (see legend). Filled and empty symbols correspond to semiflexible and flexible rings respectively. Solid and dashed lines are guides for the eyes. The vertical dotted line indicates the overlap density, $\rho_{\text {eff }}=1$.

particles, with extremely high packing fractions that are impossible in atomic liquids. This is a direct consequence of the highly penetrable character of the rings. Indeed the overlap density of the effective fluid, $\rho_{\text {eff }}=1$, actually corresponds to monomer densities that are far from melt conditions $(\rho \approx 1)$. Thus, at the overlap density $\rho_{\text {eff }}=1$ the monomer densities range from $\rho \approx 0.01$ (semiflexible rings of $N=100$ ) to $\rho \approx 0.30$ (flexible rings of $N=20)$. Therefore, in all the investigated systems the monomer concentration can be largely increased to drive the corresponding effective fluid far beyond the overlap density.

Figure 3 shows the effect of the density on the average diameter of gyration of the rings. For the same molecular weights, we include data of the semiflexible rings investigated in this work and data of the flexible rings investigated in Ref. [44]. To facilitate the comparison between different data sets, the diameters of gyration are normalized by their respective values at infinite dilution. Thus, this representation allows to compare the relative changes 
of the ring size in all the investigated systems. As discussed in Ref. [44], flexible rings exhibit a clear shrinkage by increasing density. This is reflected in the monotonic decrease of $D_{\mathrm{g}}$ by varying $\rho_{\text {eff }}$ from zero to the highest investigated value. The reduction of $D_{\mathrm{g}}$ is already significant (about a $5 \%$ ) at the overlap density and it becomes much more pronounced beyond it, even reaching a $37 \%$ at the highest investigated density for $N=100$. The introduction of stiffness through the bending potential of Eq. (3) leads to a rather different density dependence of the ring size. The semiflexible rings show a much weaker shrinkage than their flexible counterparts. Thus, at the highest investigated effective densities of the flexible rings, $D_{\mathrm{g}} / D_{\mathrm{g} 0}=0.81,0.68,0.63$ for $N=20,50$, and 100 respectively. At the same effective densities, the corresponding values in the semiflexible rings are $D_{\mathrm{g}} / D_{\mathrm{g} 0}=0.995$, 0.95 and 0.90. Having noted this, the data for the semiflexible rings in Fig. 3 reveal a novel feature: the presence of a minimum in $D_{\mathrm{g}} / D_{\mathrm{g} 0}$ at $\rho_{\text {eff }} \sim 8$ for $N=50$ and at $\rho_{\text {eff }} \sim 16$ for $N=100$, followed by a further increase of the ring size at higher densities. Amazingly, for the case $N=50$ at the highest investigated density, the semiflexible rings expand so much that their size becomes larger (by a $5 \%$ ) than in the limit of high dilution. Because of the much higher computational cost, in comparison to the case of flexible rings, of simulating higher densities for the semiflexible rings, we cannot confirm whether this feature $\left(D_{\mathrm{g}} / D_{\mathrm{g} 0}>1\right)$ will also be observed for $N=20$ and $N=100$ (indeed for $N=20$ we have not even observed the minimum in $\left.D_{\mathrm{g}} / D_{\mathrm{g} 0}\right)$.

At this point we have to stress that the anomalous density dependence of $D_{\mathrm{g}}$ is a specific feature of the semiflexible rings. It will not be observed for flexible rings by exploring higher densities, since indeed the much less demanding simulations of flexible rings have already accessed melt densities (see Fig. 2). The rather different trends observed for semiflexible and flexible rings in Fig. 3 can be rationalized as follows. The shrinkage of flexible rings upon compression has an entropic origin, since there is no energetic cost over open configurations due to the absence of bending energy. However, shrinking of the semiflexible rings involves a strong increase of the bending energy and is highly unfavourable. In these conditions the free energy is reduced by adopting energetically favourable open conformations and, as we will show in next sections, interpenetration and even clustering of the rings in order to fill the space. 


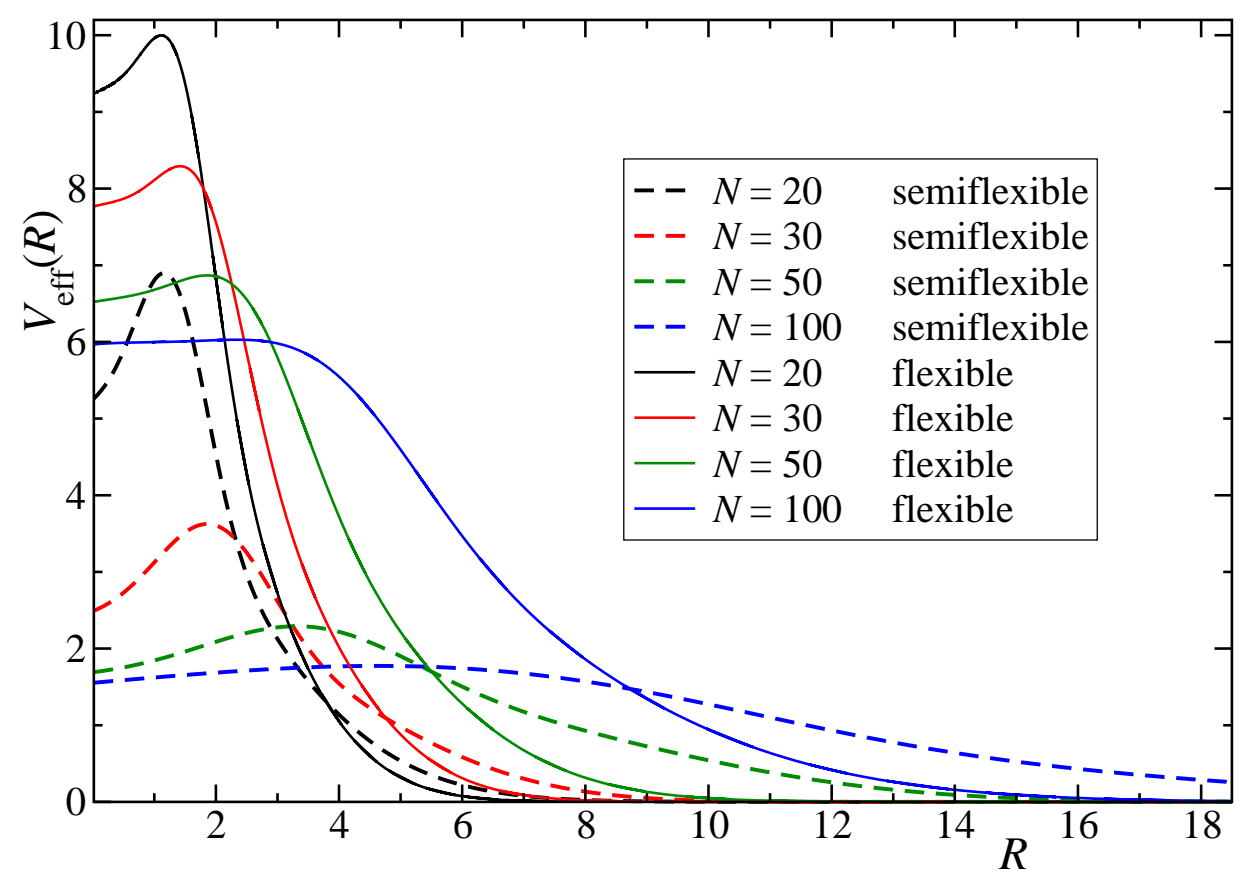

FIG. 4: Effective potentials at infinite dilution for semiflexible (dashed lines) and flexible rings (solid lines). Data sets for a same molecular weight are represented with identical colours (see legend). Unlike in Figs. 6 - 7 (see below), the distance $R$ is not normalized by $D_{\mathrm{g} 0}$, but is given in units of the 'monomer size' $\sigma$.

\section{B. Effective potentials}

Fig. 4 displays the effective potentials $V_{\text {eff }}(R)$ obtained, by umbrella sampling MC, for the semiflexible rings at different values of $N$. We include analogous results for the flexible rings with the same $N$, taken from Ref. [44]. The comparison between the data sets of the semiflexible and flexible rings reveals a strong effect of the intramolecular barriers on the effective potentials. Thus, at fixed $N$ the interaction range of $V_{\text {eff }}(R)$ extends over longer distances for the semiflexible rings than for the flexible ones. This result is consistent with the larger size of the semiflexible rings (Fig. 1), allowing for contacts at larger separations. On the other hand, the effective potentials of the semiflexible rings have lower amplitudes than those of the flexible counterparts. This feature can be tentatively understood as follows: because of the larger size of the semiflexible rings, the monomers are distributed over a larger volume than in the flexible counterparts with the same $N$. This makes contacts between the monomers of the two rings less frequent, leading to a lower cost in free energy and favouring 


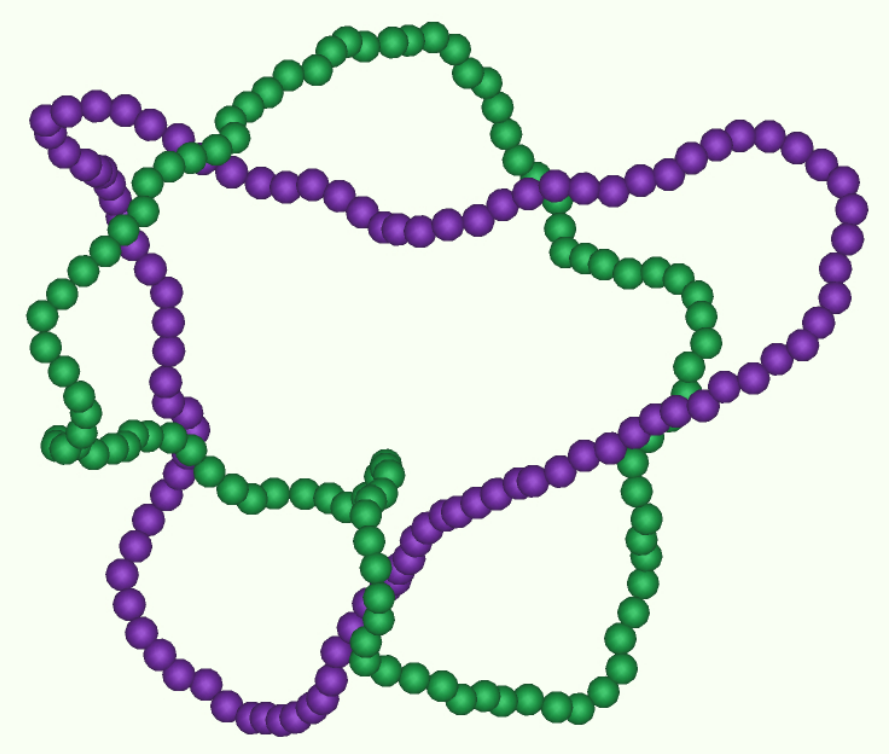

FIG. 5: Typical snapshot of two interpenetrating semiflexible rings of $N=100$ with coinciding centers-of-mass.

interpenetration.

The drop of $V_{\text {eff }}(R)$ from the maximum to the minimum at $R=0$ is steeper for the semiflexible rings than for the flexible rings with the same $N$. As discussed in Ref. [44] for the flexible rings, the minimum at $R=0$ reflects the fact that, in terms of free energy, the coincidence of the centers-of-mass $(R=0)$ is favoured over penetration with no coincidence $(R>0)$. This is due to the fact that typical configurations of isolated flexible rings at small separation consist of one ring adopting an open conformation, which provides free space and facilitates full interpenetration by the other ring [44]. This effect is enhanced in the case of semiflexible rings, for which open conformations are inherent (see a typical snapshot in Fig. 5). On the other hand, as the molecular weight increases the low- $R$ range of $V_{\text {eff }}(R)$ flatens (see Fig. 4). This feature reflects that for sufficiently large rings strong interpenetration does not involve frequent contacts between monomers. In that situation the specific distance between centers-of-mass does not affect significantly the cost in free energy.

The analysis of the effective potentials for the flexible rings [44] suggested an amplitude $V_{\text {eff }}(R=0) \sim 6 k_{\mathrm{B}} T$ in the limit of high molecular weight. We expect that the amplitude for the semiflexible rings will converge to this value for large $N$, since specific effects of 
semiflexibility should vanish in that limit. If this is the case, the amplitude of the effective potential of the semiflexible rings will behave in a non-monotonic fashion. Indeed we find $V_{\text {eff }}(R=0) \lesssim 2 k_{\mathrm{B}} T$ for the highest investigated $N$ (see Fig. 4). The expected increase of $V_{\text {eff }}(R=0)$ up to the limit $\sim 6 k_{\mathrm{B}} T$ by increasing $N$ can be tentatively understood as follows. In the limit of large $N$, the statistics of the self-avoiding random walk will be recovered at long intramolecular distances. As in the case of the flexible rings, this will facilitate folding of portions of the semiflexible rings - e.g. by adopting 8-like configurations that are still energetically very unfavourable for the largest semiflexible rings investigated here, $N=100$. Likewise, open configurations of the rings will be less frequent for $N \gg 100$, and then interpenetration will be more strongly hindered by contacts between monomers. This effect will lead to an effective increase of the free energy cost for full interpenetration. Unfortunately, the approach to the limit $V_{\text {eff }}(R=0) \sim 6 k_{\mathrm{B}} T$ could only be confirmed by performing rather demanding simulations of semiflexible rings with higher $N$ than those investigated here.

\section{Effective fluid vs. real system}

As discussed in previous works [44, 47,61], a direct consequence of the observed features of $V_{\text {eff }}(R)$ in the low- $R$ region is that the Fourier transform (FT) of the potential has negative components, i.e, the potential belongs to the so-called $Q^{ \pm}$-class (potentials with non-negative FT are termed $Q^{+}$-class) [62]. We have checked that all potentials of Fig. 4 belong to the

$Q^{ \pm}$-class by explicitly analyzing their FT's. A robust argument based on density functional theory (DFT) [62] establishes that if a bounded potential belongs to the $Q^{ \pm}$-class it will form a cluster crystal phase at high densities [62-68], which is anticipated at lower densities by a strongly clustered, disordered fluid phase. Having noted this, we investigate the limits of validity, at finite concentrations, of the obtained effective potentials and in particular the possible realization of clustering in the real system of semiflexible rings. For this, we compare MD simulations of the real system and the corresponding effective fluid of ultrasoft particles. The interactions between monomers in the real fluid are given by the potentials of Eqs. (13 ), whereas the ultrasoft particles interact through the effective potentials, $V_{\text {eff }}(R)$, obtained at infinite dilution by $\mathrm{MC}$ umbrella sampling. We have computed the radial distribution function $g(R)$ of the centers-of-mass of the fluid of real rings and compared it with the 


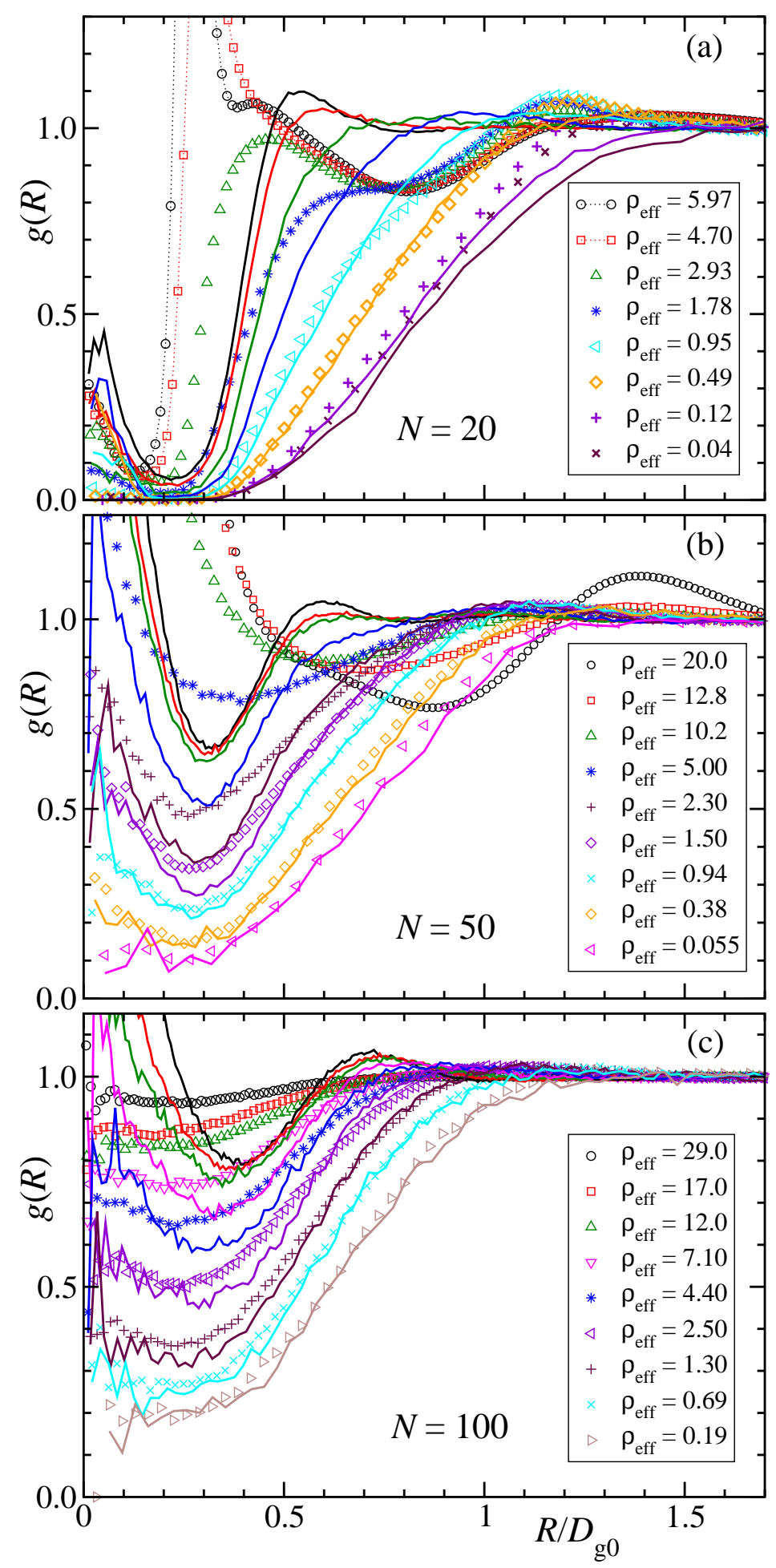

FIG. 6: Radial distribution functions of the centers-of-mass for the semiflexible rings of length $N=20$ (a), 50 (b) and 100 (c). Distances are normalized by the respective infinite-dilution diameters of gyration. Symbols: simulation results for the real system. Lines: simulation results for the effective fluid with the infinite-dilution effective pair potential $V_{\text {eff }}(R)$. 


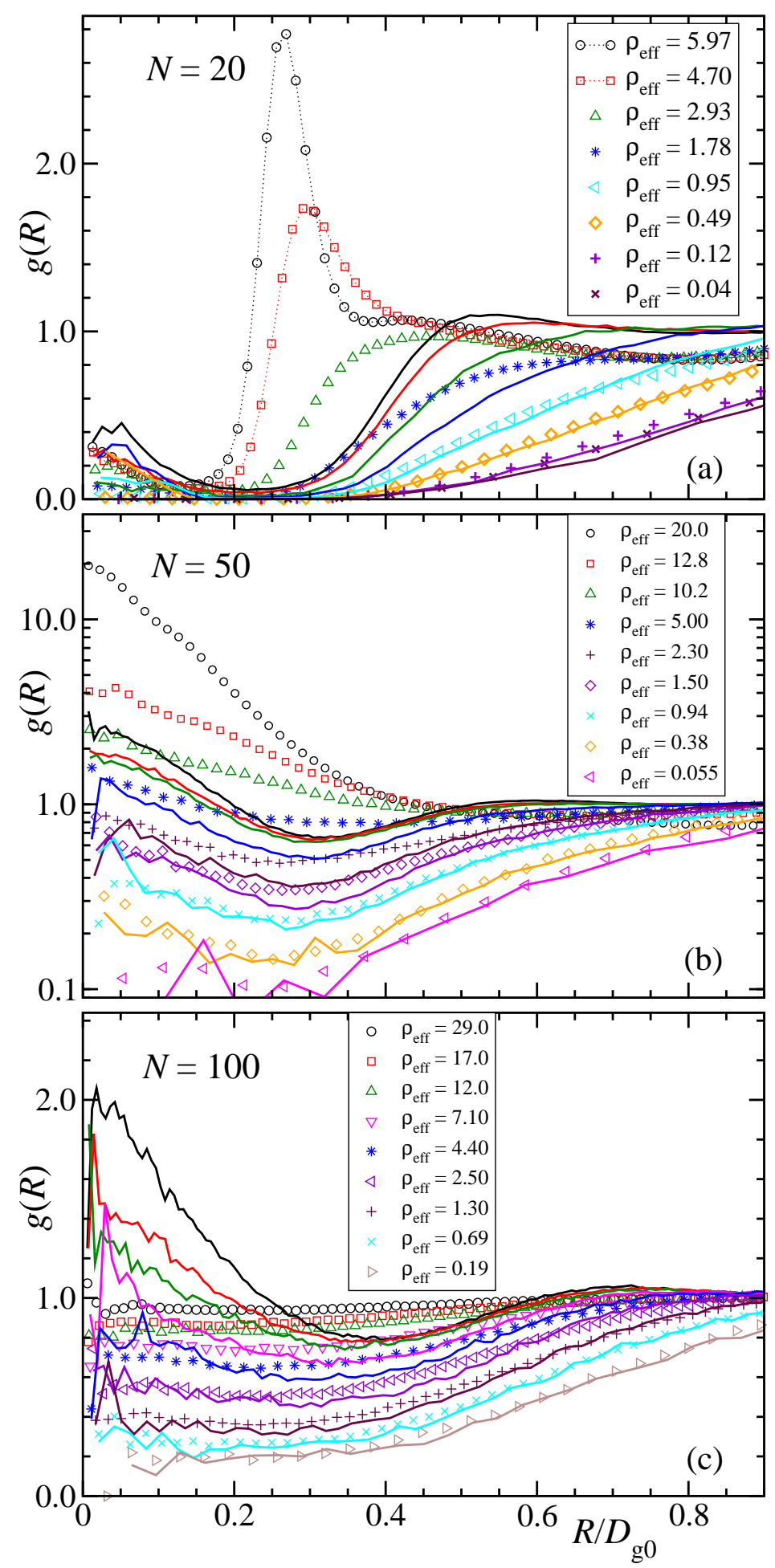

FIG. 7: Same data of Fig. 6 represented in a different scale, in order to facilitate visualization of clustering at high densities. Note the logarithmic scale for $g(R)$ in panel (b). 
respective $g(R)$ of the effective fluid. Figs. 6 and 7 show this comparison for several values of $N$ and at different effective densities. Both figures display the same data sets, though in different scales in order to facilitate visualization of several salient features of $g(R)$. Distances are normalized by the respective values of the infinite-dilution diameter of gyration $D_{\mathrm{g} 0}$.

Inspection of the data of Figs. 6 and 7 reveal some common trends and some differences between the different investigated systems. Because of the bounded character of the effective potentials the ultrasoft particles can be fully interpenetrated, and for sufficiently high densities the $g(R)$ of the effective fluid exhibits a sharp maximum at $R=0$ and a minimum at small separations $R<0.4 D_{\mathrm{g} 0}$. This feature reflects clustering of the ultrasoft particles and is consistent with the specific features of the effective potentials discussed above. Though the clustering behaviour of the effective fluid is evident, we have to make clear that the spatial arrangement of the ultrasoft particles is amorphous. The cluster crystal phase was not formed at any of the investigated densities and molecular weights. This was confirmed by visual inspection of snaphots of the effective fluid, and indeed is consistent with the expected locus of the freezing lines of the effective fluids. Though a precise determination of such lines - e.g. by combination of simulation and DFT as in Ref. [63] — is beyond the scope of this work, a qualitative underestimation can be obtained by the relation $\rho_{\text {eff }}=T D_{g 0}^{3} /\left(1.393\left|V^{*}\right|\right)$, with $V^{*}$ the FT of the effective potential at its absolute minimum [64]. From this relation we find the freezing densities $\rho_{\text {eff }} \approx 20,39$ and 38 for the cases $N=20,50$ and 100 respectively. These values are above the highest investigated densities for each $N$ (see Fig. 2), and therefore cluster crystals are not found in the simulations.

For a same density (see data in Fig. 6 for e.g., $\rho_{\text {eff }} \sim 5$ ), the minimum in the $g(R)$ of the effective fluid is more pronounced for smaller $N$. In particular for $N=20$ at the highest investigated densities, the maxima at $R=0$ and $R \sim 0.5 D_{\mathrm{g} 0}$ are separated by a strong correlation hole. This can be understood as a consequence of the shape of the effective potential for $N=20$ (Fig. 4). The height of the maximum, $V_{\mathrm{eff}} \approx 7 k_{\mathrm{B}} T$, is considerable and strongly hinders full interpenetration, leading to the correlation hole in $g(R)$. However, the minimum of $V_{\text {eff }}$ at $R=0$ is a sufficient condition for having negative components in the FT $[44,47,61]$, and hence for clustering behaviour, which leads to the maximum at $R=0$ in $g(R)$.

Now we discuss to which extent the former features are confirmed in the fluid of real semiflexible rings. Thus, for rings of $N=50$ and $N=100$ the effective fluid provides 
an excellent description of the structure of the real system at concentrations below the overlap density. However for the smallest investigated rings, $N=20$, this description is at best semiquantitative and a significant discrepancy between the effective and the real $g(R)$ is found. This is the case even at concentrations far below the overlap density (see, e.g., data for $\rho_{\text {eff }}=0.04$ in Fig. 6a), for which effective potentials generally provide an accurate description of the real fluid $[28,29]$. As we will discuss in Section IV, the mentioned discrepancy at high dilution can be tentatively related to the fact that the used effective potentials do not incorporate orientational degrees of freedom, which may be very relevant for the specific system investigated here.

As the concentration increases above the overlap density, the description by the effective fluid progressively worsens for all values of $N$. Still, for $N=50$ and $N=100$ a semiquantitative agreement between the real and effective $g(R)$ is found at densities up to $\rho_{\text {eff }} \sim 2$. By further increasing the density strong discrepancies between the effective and real $g(R)$ are evident. At such high densities the contribution of many-body forces becomes more and more relevant, and the effective potential obtained at high dilution is no longer a good representation of the interactions. Having said this it is noteworthy that, with different degrees of accuracy depending on the investigated density, the description in terms of the effective fluid accounts for a main feature: the introduction of intramolecular stiffness favours interpenetration and clustering in the real system of semiflexible rings. This is confirmed by the maximum at $R=0$ in the real $g(R)$ and reproduced, at least qualitatively, by the effective fluid.

This was not the case for the flexible rings investigated in Ref. [44]. The effective potentials derived at high dilution were no longer valid for the density range relevant for clustering, and indeed the clustering behaviour displayed by the effective fluid was not observed at any density in the real system of flexible rings. This clear discrepancy was assigned to the progressive shrinking of the flexible rings with increasing concentration. This effect prevented clustering and led to a strong correlation hole in the $g(R)$. On the contrary, semiflexible rings weakly shrink and even swell with increasing density (Fig. 3). Hence they have to interpenetrate in order to fill the space, which leads to the formation of clusters. 


\section{DISCUSSION}

In the previous section we have shown that the effective interactions between semiflexible rings obtained at high dilution can account, at least qualitatively, for interpenetration and clustering of the rings in concentrate solutions. In this section we characterize in detail the specific features of the clustering behaviour for the different investigated molecular weights. The strength of the clustering, which is reflected by the height of the maximum of the real $g(R)$ at $R=0$, is strongly dependent on both density and $N$. The most pronounced effects are observed for semiflexible rings of $N=50$, which already form clusters at concentrations below the overlap density (see data for $\rho_{\text {eff }} \sim 0.4$ in Fig. $6 \mathrm{~b}$ ), and show a value $g(R=0) \approx 20$ at the highest investigated density (Fig. 7b).

For rings of $N=100$, clustering is observed above the overlap density, though in this case it is a minor feature (Fig. 6c). This is reflected by the small difference between the maximum at $R=0$ and the first minimum in $g(R)$. Clustering for $N=100$ tends to vanish at the highest investigated densities. Still, the data evidence a full interprenetration of the rings and the results approach the ideal gas limit $g(R)=1$, reflecting a nearly random distribution of the centers-of-mass. This is the typical behaviour of ultrasoft particles of the $Q^{+}$-class in the limit of high density [69], in contrast to the clustering expected for the effective potential of the $Q^{ \pm}$-class obtained at high dilution. This feature clearly reflects the increasing strength of the many-body contributions at high densities, which fully alter the nature of the effective interaction between the rings.

The $g(R)$ of the semiflexible rings of $N=20$ displays complex features. Clustering is present at the highest investigated densities, as reflected by the maximum of $g(R)$ at $R=0$ (Fig. 6a). However, the main structural feature is given by a prominent peak at finite separation $\left(R<0.3 D_{\mathrm{g} 0}\right.$ for $\rho_{\mathrm{eff}}>4$, see Fig. 7a). Though the weak maximum at $R=0$ shows that full interpenetration is not forbidden, the presence of such a strong peak at finite $R$ reveals that the former is unfavourable.

In the next subsections we show that the differences observed in the clustering behaviour for the different investigated molecular weigths can be understood by characterizing the typical intramolecular conformations of the rings, as well as their relative orientations. The analysis will reveal relevant anisotropic features that definitively cannot be captured by the effective potentials, suggesting the need to improve the standard approach by adding the 
relative orientations as relevant coordinates for the effective interactions.

\section{A. Shape parameters}

We have characterized the shape of the semiflexible rings by analyzing the asphericity and prolateness parameters [70-74]. These can be obtained from the radius of gyration tensor,

$$
T_{\alpha \beta}=\frac{1}{N^{2}} \sum_{i=1}^{N}\left(r_{i \alpha}-r_{\alpha}^{\mathrm{cm}}\right)\left(r_{i \beta}-r_{\beta}^{\mathrm{cm}}\right),
$$

where $\alpha, \beta$ denote cartesian components. The vectors $\mathbf{r}_{i}$ and $\mathbf{r}^{\mathrm{cm}}$ are the positions of the monomers and the center-of-mass of the rings, respectively. In the following we sort the three eigenvalues of the tensor as $\lambda_{1} \leq \lambda_{2} \leq \lambda_{3}$. It can be seen that $D_{\mathrm{g}}^{2}=4\left(\lambda_{1}+\lambda_{2}+\lambda_{3}\right)$. The asphericity parameter, $0 \leq a \leq 1$, is defined as:

$$
a=\frac{\left(\lambda_{2}-\lambda_{1}\right)^{2}+\left(\lambda_{3}-\lambda_{1}\right)^{2}+\left(\lambda_{3}-\lambda_{2}\right)^{2}}{2\left(\lambda_{1}+\lambda_{2}+\lambda_{3}\right)^{2}} .
$$

The asphericity of a perfectly spherical object is $a=0$. Objects with approximate spherical shape $\left(\lambda_{1} \approx \lambda_{2} \approx \lambda_{3}\right)$ have asphericities $a \gtrsim 0$. The prolateness parameter, $-1 \leq p \leq 1$, is defined as:

$$
p=\frac{\left(2 \lambda_{1}-\lambda_{2}-\lambda_{3}\right)\left(2 \lambda_{2}-\lambda_{1}-\lambda_{3}\right)\left(2 \lambda_{3}-\lambda_{1}-\lambda_{2}\right)}{2\left(\lambda_{1}^{2}+\lambda_{2}^{2}+\lambda_{3}^{2}-\lambda_{1} \lambda_{2}-\lambda_{1} \lambda_{3}-\lambda_{2} \lambda_{3}\right)^{3 / 2}} .
$$

For perfectly oblate objects $\left(\lambda_{1}<\lambda_{2}=\lambda_{3}\right)$ the prolateness is $p=-1$. For perfectly prolate objects $\left(\lambda_{1}=\lambda_{2}<\lambda_{3}\right) p=1$.

Figs. 8 and 9 show normalized results for the distribution of asphericities, $P(a)$, and prolateness, $P(p)$, respectively [75]. The data are given for $N=20,50$, and 100 at different concentrations, from infinite dilution to the highest investigated density. Lines and symbols are data for $\rho_{\text {eff }}$ below and above the overlap density, respectively. The distributions are very weakly affected by increasing the density up to the overlap concentration. This is consistent with the observations for the diameter of gyration (see Fig. 3). Therefore, we can conclude that both the size and shape of the semiflexible rings are essentially unperturbed for densities up to the overlap concentration.

The maxima of $P(a)$ are in the range $0.10<a<0.18$. Whereas $P(a)$ is approximately symmetrical for $N=50$, it shows a strong broadening in the low- and high- $a$ range for $N=20$ and $N=100$ respectively. The comparison between the $P(a)$ of the three selected 


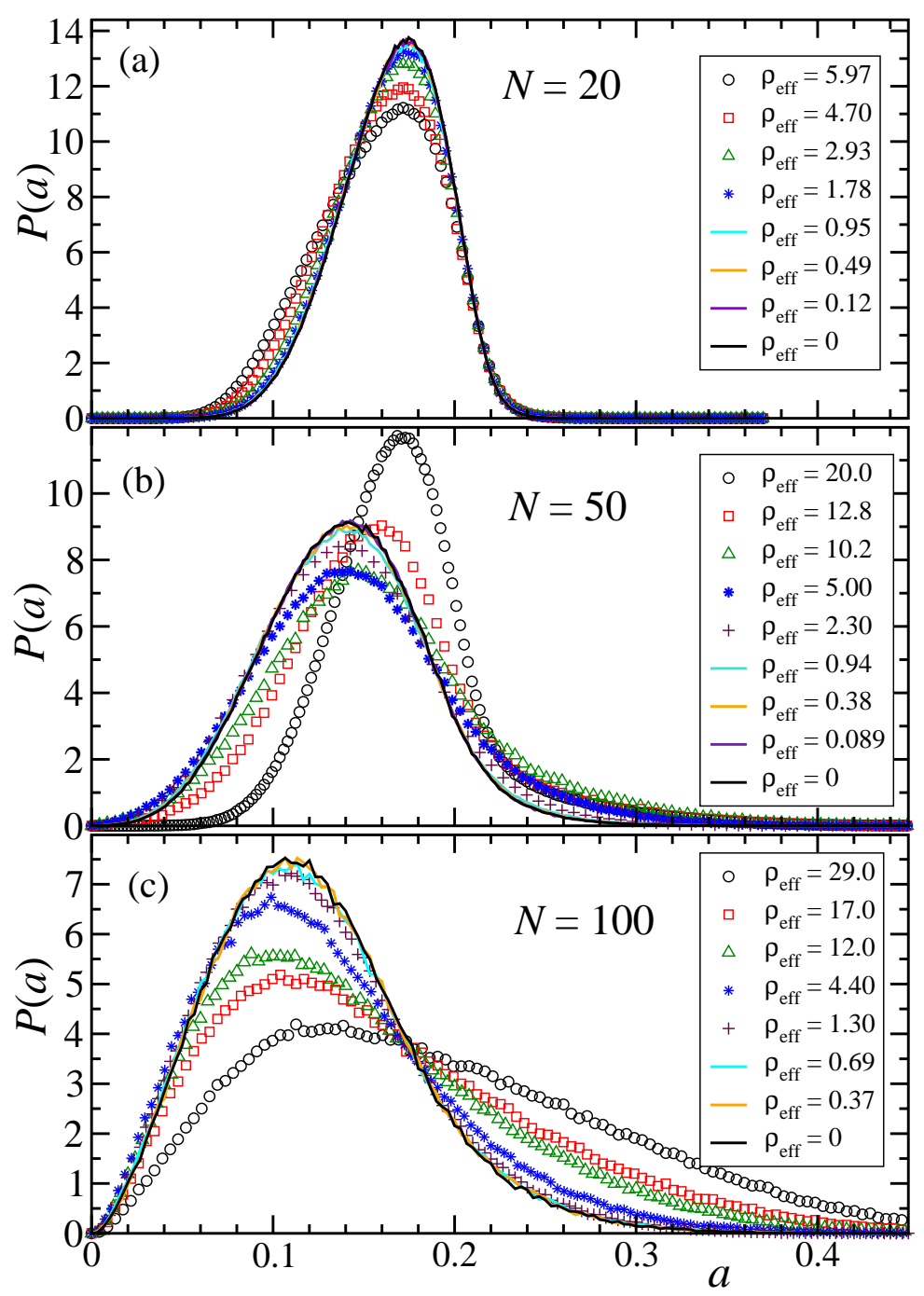

FIG. 8: Distribution of asphericity for the semiflexible rings of molecular weight $N=20$ (a), 50 (b) and 100 (c). Different data sets (see legends) correspond to different densities below (lines) and above (symbols) the overlap density.

values of $N$ reveals a pronounced ability for larger rings to adopt different shapes. Instead, the asphericity of the smallest investigated rings $(N=20)$ is restricted to a narrow interval, and the probability of adopting nearly spherical shapes $(a \rightarrow 0)$ is negligible. This can be easily understood by visual inspection of the rings, which for $N=20$ adopt disk-like conformations at all densities (see below). Obviously, such conformations do not fulfill the necessary condition $\lambda_{1} \approx \lambda_{2} \approx \lambda_{3}$ for spherical character. The disk-like character of the rings of $N=20$ is also reflected in the distribution of prolateness $P(p)$, which is monotonic and clearly dominated by values $p \rightarrow-1$ (see Fig. 9a), i.e., by oblate configurations. 

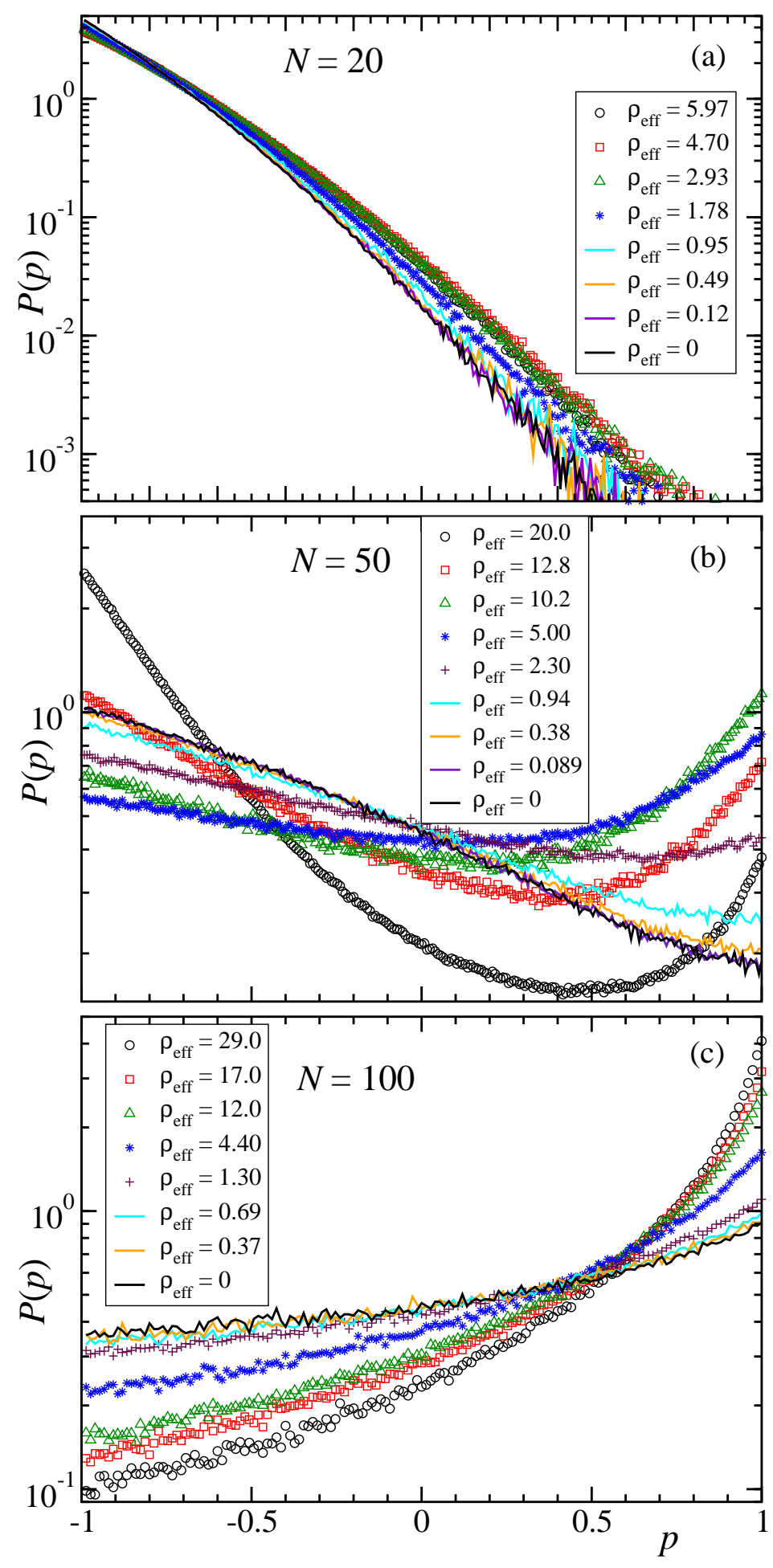

FIG. 9: Distribution of prolateness for the semiflexible rings of molecular weight $N=20$ (a), 50 (b) and 100 (c). Different data sets (see legends) correspond to different densities below (lines) and above (symbols) the overlap density. 
The distributions $P(a)$ and $P(p)$ for $N=50$ and $N=100$ exhibit strong changes when the density is increased beyond the overlap concentration. This effect is much less pronounced for the case $N=20$, which again reflects the almost underformable character of the semiflexible rings in the limit of low $N$. By increasing $\rho_{\text {eff }}$ beyond the overlap density the distribution $P(a)$ for $N=100$ exhibits a progressive broadening and shift to large $a$-values, i.e., the rings become less and less spherical. Concomitantly, the distribution $P(p)$ is more and more dominated by prolate objects. A more complex behaviour is revealed for $N=50$. At densities below and around the overlap concentration $P(p)$ is largely dominated by oblate objects and displays a monotonic $p$-dependence. However, a strong non-monotonic character arises for densities $\rho_{\text {eff }}>2.3$. At such densities $P(p)$ exhibits two maxima in the limits of oblate $(p=-1)$ and prolate $(p=1)$ objects. The bimodal character of $P(p)$ becomes more pronounced by further increasing the density. The distribution is extremely bimodal at the highest investigated $\rho_{\mathrm{eff}}=20$. Still, the distribution is not symmetric but dominated by oblate objects.

\section{B. Relative orientation}

In this subsection we characterize orientational correlations between neighbouring rings at high concentrations. For each ring we define a 'normal vector' as $\mathbf{u}=\sum_{i} \mathbf{b}_{i} \times \mathbf{b}_{i+1}$, where the sum is done over all pairs of consecutive bonds, with $\mathbf{b}_{i}$ and $\mathbf{b}_{i+1}$ the corresponding bond vectors. By using their normal vectors $\mathbf{u}$ and $\mathbf{u}^{\prime}$, we can characterize the relative orientation between two rings by the angle $\theta=\cos ^{-1}(s)$, with $s=\mathbf{u} \cdot \mathbf{u}^{\prime} /\left|\mathbf{u} \| \mathbf{u}^{\prime}\right|$. For angles $\theta>90^{\circ}$ we do the transformation $\theta \rightarrow 180^{\circ}-\theta$, so that relative orientations are defined in the range $0^{\circ} \leq \theta \leq 90^{\circ}$. We compute the distribution $P(\theta)$ for all the pairs of 'neighbouring' rings $j, k$. Neigbouring rings are defined as those for which the distance $R_{j k}$ between their centers-of-mass is smaller than some value $R_{\mathrm{c}}$. The latter should be some fraction of $D_{\mathrm{g} 0}$ to probe ring interpenetration, but should not be too small in order to get good statistics. In the following we will present results for the case $R_{\mathrm{c}}=0.6 D_{\mathrm{g} 0}$. Other moderate fractions of $D_{\mathrm{g} 0}$ provide the same qualitative features for $P(\theta)$.

Fig. 10 shows results of $P(\theta)$ for $N=20,50$, and 100 at different concentrations, from infinite dilution to the highest investigated density. Within statistics, all data sets for $N=100$ overlap, i.e, the relative orientation between neighbouring rings of $N=100$ 

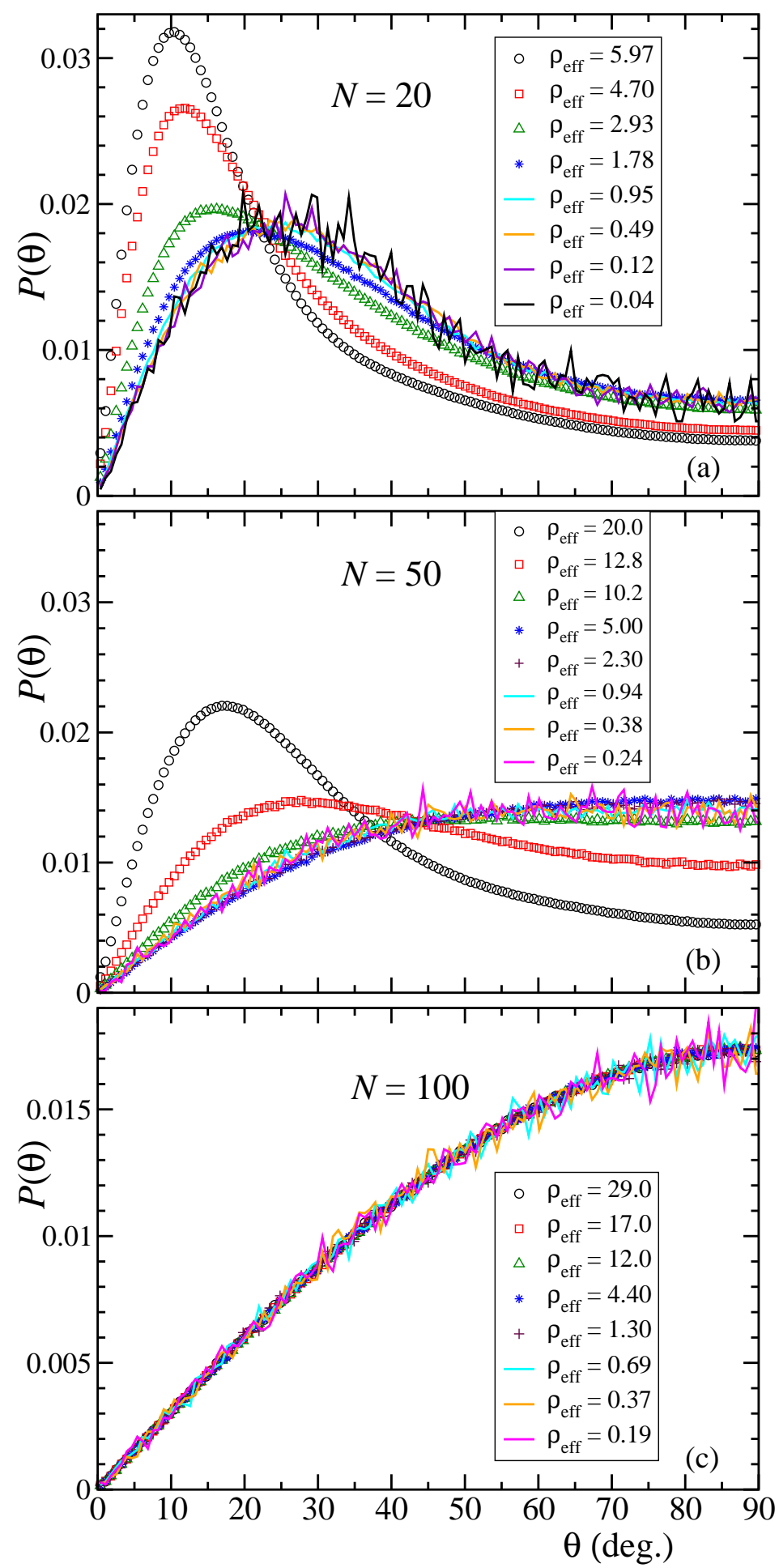

FIG. 10: Distribution of relative orientations for the semiflexible rings of molecular weight $N=20$ (a), 50 (b) and 100 (c). Different data sets (see legends) correspond to different densities below (lines) and above (symbols) the overlap density. 


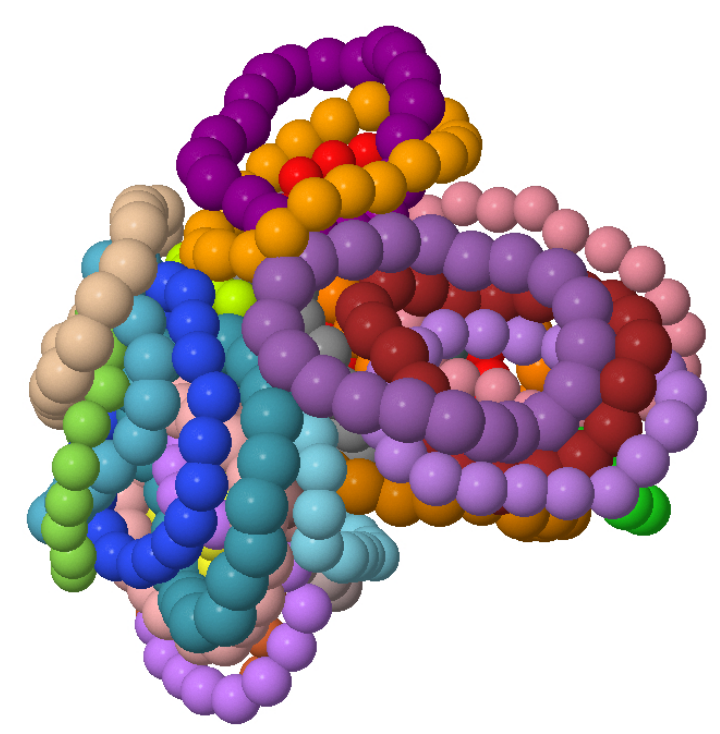

FIG. 11: For effective density $\rho_{\text {eff }}=5.97$, snapshot of a semiflexible ring of $N=20$ and all its neigbours at $R<D_{\mathrm{g} 0}$. Different rings are represented with different colours in order to facilitate visualization of local discotic order.

is independent of the concentration. The distribution $P(\theta)$ is dominated by high angles. Thus, $50 \%$ of the angles are $\theta>60^{\circ}$, and quasi-parallel orientations $(\theta \rightarrow 0)$ are very rare. On the contrary, the relative orientations between neighbouring rings of $N=20$ are preferentially quasi-parallel. The distribution $P(\theta)$ is unperturbed for densities below the overlap concentration, with a maximum at $\theta \approx 25^{\circ}$. The $50 \%$ of the angles are $\theta<35^{\circ}$. By increasing the density beyond the overlap concentration the distribution shifts to the low- $\theta$ range. At the highest investigated density it shows a maximum at $\theta \approx 10^{\circ}$, and the $50 \%$ of the angles lie in the region $\theta<20^{\circ}$.

The quasi-parallel character of the relative orientations between neighbouring rings of $N=20$ is confirmed by visual inspection. Fig. 11 shows a typical snapshot of a portion of the simulation cell at the highest investigated density. The figure represents a ring and all its neighbours within a distance $R<D_{\mathrm{g} 0}$. The figure confirms the disk-like conformations suggested by the analysis of the asphericity and prolateness (see Figs. 8 and 9). The rings arrange in small domains with local discotic order that is lost at distances of a few times the ring size. This clarifies the origin of the sharp peak at finite $R$ in the $g(R)$ (see data for $\rho_{\text {eff }} \geq 4.70$ in Fig. 7a). Such a peak arises from the excluded volume induced by the 
monomers in the quasi-parallel allignement of the neighbouring rings. Note that indeed the position of the peak, at $R \lesssim 0.3 D_{\text {g0 }}$, just corresponds to $R \lesssim 1.8$ in units of $\sigma$ (the bead diameter). This reflects the typical distance between the centers-of-masses of two nearestneighbour quasi-parallel disk-like rings. Moreover the snapshot of Fig. 11 shows that, given the small size and low deformability of the rings of $N=20$, the coincidence of centers-ofmasses - i.e., 'clustering' - is very unprobable. Still, it can happen for reduced groups of neighbouring rings, yielding the small peak at $R=0$ in $g(R)$ (see Fig. 6a).

Consistently with the complex trends found for the diameter of gyration, asphericity and prolateness (see above), the distribution $P(\theta)$ for the rings of $N=50$ exhibits a complex density dependence (Fig. 10b). In the concentration range from infinite dilution to the overlap density $P(\theta)$ is density-independent within statistics, and it is just weakly perturbed by further increasing the density up to $\rho_{\text {eff }} \sim 10$. In this range of concentrations the distribution for $N=50$ shows the same qualitative monotonic behaviour found for $N=100$, though $P(\theta)$ is steeper for $N=100$. However, for densities $\rho_{\text {eff }}>10$ the distribution evolves to adopt the qualitative aspect found for $N=20$. Thus, at these densities the relative orientations between neighbouring rings of $N=50$ are preferently quasi-parallel. For the highest investigated $\rho_{\mathrm{eff}}=20,50 \%$ of the angles lie in the domain $\theta<30^{\circ}$. In spite of this analogy with the corresponding distribution $P(\theta)$ for $N=20$, we found in the analysis of $g(R)$ a clustering behaviour for $N=50$ rather different from that observed for $N=20$. Namely, $g(R)$ shows a sharp peak at $R=0$ (see Fig. 7b) and no correlation hole. The amplitude of the peak is a clear signature of strong clustering, rather different from the minor effect found for rings of $N=20$.

Now we clarify the nature of the clustering behaviour in the rings of $N=50$. Fig. 12 shows a typical snapshot of the centers-of-mass of the rings at the highest investigated density $\rho_{\text {eff }}=20$. We confirm that the rings do not form a cluster crystal phase - actually this was not even formed by the effective fluid at this concentration, which is below the theoretical freezing density (see above). Instead, the rings arrange in a complex disordered phase consisting of long columns of rings. Fig. 13 shows, for the same density, a partial snapshot of the monomers. The figure displays a ring and all its neighbours within a distance $R<0.7 D_{\mathrm{g} 0}$. The snapshot reveals that the columns observed in Fig. 12 are partially formed by quasi-parallel rings. This is consistent with the observation of dominant low angles in the distribution $P(\theta)$ (see Fig. 10b). Moreover, the columns are fully penetrated by bundles of 


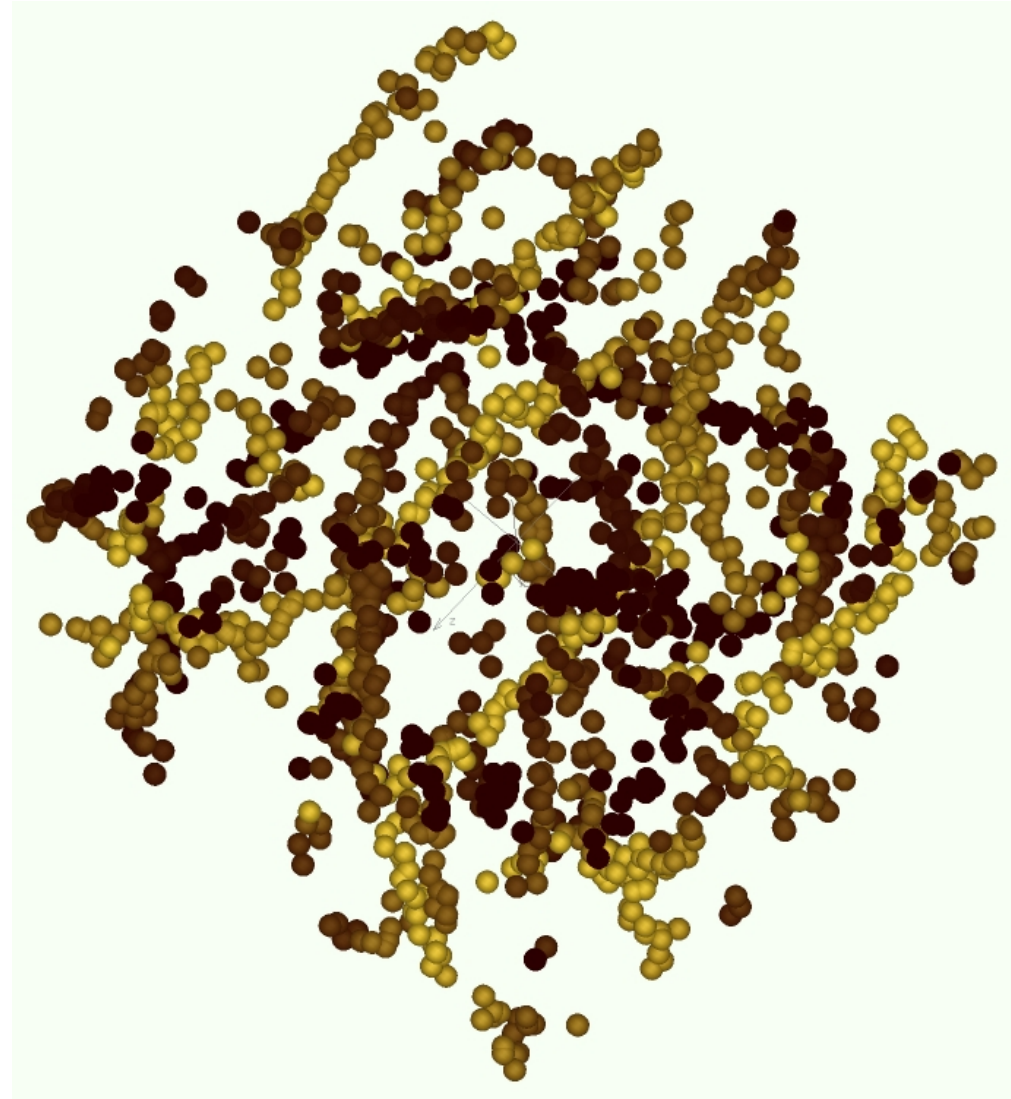

FIG. 12: Snapshot of the centers-of-mass of the semiflexible rings of $N=50$ at effective density $\rho_{\text {eff }}=20.0$. Depth cueing is used to facilitate visualization of columns.

elongated rings. Fig. 13 also reveals the origin of the bimodal distribution of the prolateness for $N=50$ at high densities (see Fig. 9b). Thus, the rings forming the columns are mostly oblate objects, whereas the bundles penetrating the columns are formed by elongated prolate rings.

In Section III we showed that the approach of the effective fluid is able to predict strong clustering of the rings of $N=50$, and to account for the observed trends in $g(R)$ (at worst to a qualitative level, see Fig. 7b). However, the results presented in this section demonstrate that the former approach is clearly incomplete. Obviously the used effective potentials, which only depend on the separation between centers-of-mass, cannot account for the short and middle-range anisotropy found in the real systems of semiflexible rings. In particular, such potentials cannot reproduce the long columnar structures observed for $N=50$ (Fig. 12) and, as expected, the corresponding effective fluid shows isotropic disorder - this is confirmed 


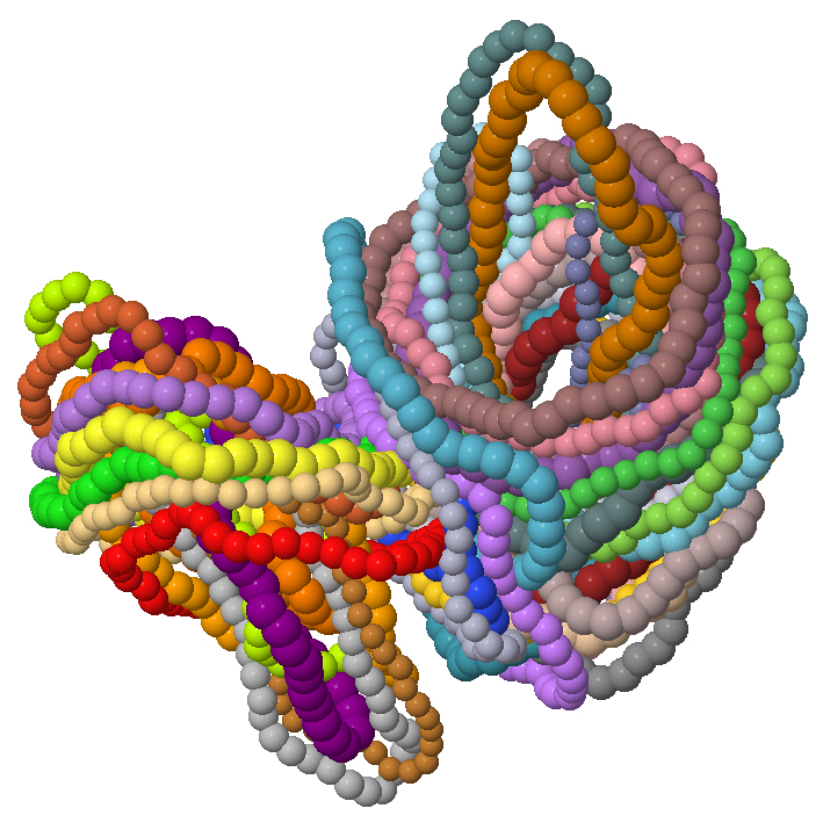

FIG. 13: For effective density $\rho_{\text {eff }}=20.0$, snapshot of a semiflexible ring of $N=50$ and all its neigbours at $R<0.7 D_{\mathrm{g} 0}$. Different rings are represented with different colours, in order to facilitate visualization of columns of oblate rings penetrated by bundles of elongated prolate rings.

by visual inspection of snapshots of the effective fluid at the same density (not shown).

Tentatively, a way of improving the description by the effective fluid would be to formulate the effective pair potential not only in terms of the separation between centers-of-mass $R$, but also including the relative orientation $\theta$ between the rings. At this point we remind that for the semiflexible rings of $N=20, V_{\text {eff }}(R)$ did not provide an accurate quantitative description of $g(R)$ even at high dilution (see Fig. 6a), i.e., in the situation for which the use of a pairwise effective potential (which is derived for two isolated macromolecules) is, in general, fully justified. Because of the small size and low deformability of the semiflexible rings of $N=20$, it seems plausible that the free energy cost for close contact of two isolated rings is strongly dependent on the relative orientation. Indeed for such small and stiff rings, contact between monomers can be avoided by quasi-parallel allignement and weak deformations, whereas for perpendicular orientation contact can only be prevented by unfavourable strong deformations of the rings. Clearly, $V_{\text {eff }}(R)$ involves an average over all orientations and misses these features. The derivation of effective potentials incorporating the relative orientation between the rings is beyond the scope of this article. Still, it is worth mentioning that a 
standard computation by umbrella MC methods - using simultaneously sampling windows for both the $R$ and $\theta$-coordinate - would be much more demanding than the one used in this work.

Another point to be addressed in future work is the possibility of forming an ordered cluster phase of semiflexible rings. It might be that the complex phase displayed for $N=50$ in Fig. 12 is a precursor of an ordered columnar phase, with parallel columns that would be again formed by oblate rings penetrated by bundles of elongated prolate rings. This ordered phase might arise at higher densities than those investigated here. Note that the effective density $\rho_{\text {eff }}=20$ for the system of Fig. 12 corresponds to a monomer density of $\rho \approx 0.3$ (see Fig. 2), i.e., still about a $30 \%$ of the melt density. Whether the mentioned ordered phase can be formed by approaching melt conditions is an open question. Still, we have to stress than performing simulations at higher densities than those studied in this work is computationally very demanding because of long relaxation times, and will require high parallelization.

The results obtained in this work show that forming conventional cluster crystal lattices (bcc, fcc..) of semiflexible rings is extremely complicated. If the rings are too small $(N=20)$ full interpenetration is very unfavourable and only rare small clusters can be formed. In the other limit, the arrangement of the centers-of-mass already adopts at moderate sizes $(N=100)$ the gas-like configuration expected for long polymers. Finally, in the narrow range of molecular mass between the former limits $(N=50)$, the anisotropy of the interactions is still an essential feature, which cannot be captured by the isotropic effective potentials that lead to standard cluster crystals. It might be that these could still be formed by a fine tuning of both the molecular weight and the strength of the intramolecular barriers, though at present this point is very speculative.

\section{CONCLUSIONS}

We have presented a detailed computational investigation on the structural properties of a fluid of semiflexible ring polymers. Stiffness is introduced by implementing intramolecular barriers. In contrast to the monotonic behaviour found for flexible rings, the size of the semiflexible rings can exhibit a non-monotonic dependence on the concentration. Because of the intramolecular barriers, the shrinkage of the semiflexible rings can be highly unfavourable 
beyond some density. In such conditions the rings can swell and adopt open configurations that facilitate interpenetration and clustering.

We have derived effective potentials between the centers-of-mass of the rings at infinite dilution, and have tested their validity over the whole range of concentrations. This has been established by comparing simulation results for the real solution of rings and for the fluid of ultrasoft particles interacting through the obtained effective potential. Except for very small rings, the effective fluid provides an accurate quantitative description of the structure of the real system up to the overlap concentration. It still provides a reasonable description over a considerable range of densities above the overlap concentration. In particular the clustering behaviour predicted by the effective description is found in the real system for a certain range of molecular masses.

However, the approach of the effective fluid is incomplete. A detailed characterization of the arrangement of the cluster-forming rings reveals that these can form a disordered complex phase, consisting of long columns of oblate rings that are penetrated by bundles of elongated prolate rings. The observed differences between the clustering behaviour in the isotropic effective fluid and the real system can be tentatively assigned to the anisotropic character of the interactions between small semiflexible rings. This effect is not captured by the standard approach, which only considers macromolecular centers-of-mass as the relevant coordinates for the effective potential. This suggests that effective descriptions should be improved by also including the relative orientation between the rings. Work in this direction is in progress.

\section{ACKNOWLEDGEMENTS}

We acknowledge financial support from the projects MAT2007-63681 (Spain) and IT-43607 (GV, Spain), and by the Austrian Science Fund (FWF) under Project No. P 23400-N16. We acknowledge CESCA (Barcelona, Spain) and CESGA (Santiago de Compostela, Spain) for generous allocation of CPU time.

[1] C. Micheletti, D. Marenduzzo, and E. Orlandini, Phys. Rep. 504, 1 (2011).

[2] J. des Cloizeaux, J. Physique Lett. 42, L433 (1981). 
[3] A. Y. Grosberg, A. Feigel, and Y. Rabin, Phys. Rev. E 54, 6618 (1996).

[4] J. M. Deutsch, Phys. Rev. E 59, R2539 (1999).

[5] A. Y. Grosberg, Phys. Rev. Lett. 85, 3858 (2000).

[6] R. Metzler, A. Hanke, P. G. Dommersnes, Y. Kantor, and M. Kardar, Phys. Rev. Lett. 88, 188101 (2002).

[7] A. Dobay, J. Dubochet, K. Millett, P.-E. Sottas, and A. Stasiak, Proc. Natl. Acad. Sci. U.S.A. 100, $5611(2003)$.

[8] N. T. Moore, R. C. Lua, and A. Y. Grosberg, Proc. Natl. Acad. Sci. U.S.A. 101, 13431 (2004).

[9] N. T. Moore and A. Y. Grosberg, Phys. Rev. E 72, 061803 (2005).

[10] E. Rawdon, A. Dobay, J. C. Kern, K. C. Millet, M. Piatek, P. Plunkett, and A. Stasiak, Macromolecules 41, 4444 (2008).

[11] M. L. Mansfield and J. F. Douglas, J. Chem. Phys. 133, 044903 (2010).

[12] M. D. Frank-Kamenetskii, A. V. Lukashin, and A. V. Vologodskii, Nature 258, 398 (1975).

[13] N. Hirayama, K. Tsurusaki, and T. Deguchi, J. Phys. A: Math. Theor. 42, 105001 (2009).

[14] M. Bohn and D. W. Heermann, J. Chem. Phys. 132, 044904 (2010).

[15] T. Vettorel, A. Y. Grosberg, and K. Kremer, Phys. Biol. 6, 025013 (2009).

[16] J. D. Halverson, W. B. Lee, G. S. Grest, A. Y. Grosberg, and K. Kremer, J. Chem. Phys. 134, 204904 (2011).

[17] S. F. Edwards, Proc. Phys. Soc. 91, 513 (1967).

[18] S. F. Edwards, J. Phys. A: Gen. Phys. 1, 15 (1968).

[19] M. Müller, J. P. Wittmer, and M. E. Cates, Phys. Rev. E 53, 5063 (1996).

[20] S. Brown and G. Szamel, J. Chem. Phys. 108, 4705 (1998).

[21] S. Brown and G. Szamel, J. Chem. Phys. 109, 6184 (1998).

[22] S. Brown, T. Lenczycki, and G. Szamel, Phys. Rev. E 63, 052801 (2001).

[23] M. Müller, J. P. Wittmer, and M. E. Cates, Phys. Rev. E 61, 4078 (2000).

[24] K. Hur, C. Jeong, R. G. Winkler, N. Lacevigc, R. H. Gee, and D. Y. Yoon, Macromolecules 44, 2311 (2011).

[25] J. D. Halverson, W. B. Lee, G. S. Grest, A. Y. Grosberg, and K. Kremer, J. Chem. Phys. 134, 204905 (2011).

[26] G. Tsolou, N. Stratikis, C. Baig, P. S. Stephanou, and V. G. Mavrantzas, Macromolecules 43, $10692(2010)$. 
[27] M. Kapnistos, M. Lang, D. Vlassopoulos, W. Pyckhout-Hintzen, D. Richter, D. Cho, T. Chang, and M. Rubinstein, Nat. Mater. 7, 997 (2008).

[28] C. N. Likos, Phys. Rep. 348, 267 (2001).

[29] C. N. Likos, Soft Matter 2, 478 (2006).

[30] A. A. Louis, P. G. Bolhuis, J. P. Hansen, and E. J. Meijer, Phys. Rev. Lett. 85, 2522 (2000).

[31] V. Krakoviack, J. P. Hansen, and A. A. Louis, Phys. Rev. E 67, 041801 (2003).

[32] C. N. Likos, H. Löwen, M. Watzlawek, B. Abbas, O. Jucknischke, J. Allgaier, and D. Richter, Phys. Rev. Lett. 80, 4450 (1998).

[33] A. Jusufi and C. N. Likos, Rev. Mod. Phys. 81, 1753 (2009).

[34] A. Jusufi, C. N. Likos, and H. Löwen, J. Chem. Phys. 116, 11011 (2002).

[35] S. Huißmann, R. Blaak, and C. N. Likos, Macromolecules 42, 2806 (2009).

[36] M. Ballauff and C. N. Likos, Angew. Chem. Int. Ed. 43, 2998 (2004).

[37] I. O. Götze, H. M. Harreis, and C. N. Likos, J. Chem. Phys. 120, 7761 (2004).

[38] S. Huißmann, C. N. Likos, and R. Blaak, Soft Matter 7, 8419 (2011).

[39] D. Gottwald, C. N. Likos, G. Kahl, and H. Löwen, Phys. Rev. Lett. 92, 068301 (2004).

[40] D. Gottwald, C. N. Likos, G. Kahl, and H. Löwen, J. Chem. Phys. 122, 074903 (2005).

[41] C. Pierleoni, C. Addison, J. P. Hansen, and V. Krakoviack, Phys. Rev. Lett. 96, 128302 (2006).

[42] C. Pierleoni, B. Capone, and J. P. Hansen, J. Chem. Phys. 127, 171102 (2007).

[43] B. Capone, C. Pierleoni, J. P. Hansen, and V. Krakoviack, J. Phys. Chem. B 113, 3629 (2009).

[44] A. Narros, A. J. Moreno, and C. N. Likos, Soft Matter 6, 2435 (2010).

[45] K. Kremer and G. S. Grest, J. Chem. Phys. 92, 5057 (1990).

[46] D. Frenkel and B. Smit, Understanding Molecular Simulation (Academic Press, San Diego, 1996).

[47] B. M. Mladek, G. Kahl, and C. N. Likos, Phys. Rev. Lett. 100, 028301 (2008).

[48] S. Caracciolo, A. Pelissetto, and A. D. Sokal, J. Stat. Phys. 60, 1 (1990).

[49] F. A. Escobedo and J. J. de Pablo, J. Chem. Phys. 102, 2636 (1995).

[50] R. Varela, K. Hinson, J. Arsuaga, and Y. Diao, J. Phys. A: Math. Theor. 42, 095204 (2009).

[51] M. P. Allen and D. J. Tildesley, Computer Simulation of Liquids (Oxford University Press, Oxford, 1989).

[52] J. A. Izaguirre, D. P. Catarello, J. M. Wozniak, and R. D. Skeel, J. Chem. Phys. 114, 2090 
(2001).

[53] I. T. Todorov and W. Smith, The DL_POLY_3 user manual (STFC Daresbury Laboratory, 2008).

[54] H. J. Limbach, A. Arnold, B. A. Mann, and C. Holm, Comput. Phys. Commun. 174, 704 (2006).

[55] http://espressomd.org.

[56] M. Doi and S. F. Edwards, The Theory of Polymer Dynamics (Oxford University Press, Oxford, 1986).

[57] L. J. Fetters, D. J. Lohse, and R. H. Colby, Physical Properties of Polymers Handbook (Ed.: J.E. Mark, Springer, 2007).

[58] R. Faller, F. Müller-Plathe, and A. Heuer, Macromolecules 33, 6602 (2000).

[59] M. Bulacu and E. van der Giessen, J. Chem. Phys. 123, 114901 (2005).

[60] M. Bernabei, A. J. Moreno, and J. Colmenero, Phys. Rev. Lett. 101, 255701 (2008).

[61] D. A. Lenz, B. M. Mladek, C. N. Likos, G. Kahl, and R. Blaak, J. Phys. Chem. B 115, 7218 (2011).

[62] C. N. Likos, A. Lang, M. Watzlawek, and H. Löwen, Phys. Rev. E 63, 031206 (2001).

[63] B. M. Mladek, D. Gottwald, G. Kahl, M. Neumann, and C. N. Likos, Phys. Rev. Lett. 96, 045701 (2006).

[64] C. N. Likos, B. M. Mladek, D. Gottwald, and G. Kahl, J. Chem. Phys. 126, 224502 (2007).

[65] C. N. Likos, B. M. Mladek, A. J. Moreno, D. Gottwald, and G. Kahl, Comput. Phys. Commun. 179, 71 (2008).

[66] B. M. Mladek, P. Charbonneau, C. N. Likos, D. Frenkel, and G. Kahl, J. Phys.: Condens. Matter 20, 494245 (2008).

[67] S. van Teeffelen, A. J. Moreno, and C. N. Likos, Soft Matter 5, 1024 (2009).

[68] K. Zhang, P. Charbonneau, and B. M. Mladek, Phys. Rev. Lett. 105, 245701 (2010).

[69] A. Lang, C. N. Likos, M. Watzlawek, and H. Löwen, J. Phys.: Condens. Matter 12, 5087 (2000).

[70] J. Rudnick and G. Gaspari, Science 237, 384 (1987).

[71] G. Zifferer and W. Preusser, Macromol. Theory Simul. 10, 397 (2001).

[72] K. Alim and E. Frey, Phys. Rev. Lett. 99, 198102 (2007).

[73] E. J. Rawdon, J. C. Kern, M. Piatek, P. Plunkett, A. Stasiak, and K. C. Millett, Macro- 
molecules 41, 8281 (2008).

[74] M. Bohn, D. W. Heermann, O. Lourenço, and C. Cordeiro, Macromolecules 43, 2564 (2010).

[75] Several computational works have determined accurately the average asphericity and prolateness in the limit of long isolated rings, namely $\langle a\rangle=0.246$ and $\langle p\rangle=0.53$ for random rings [72, 73], as well as $\langle a\rangle=0.255$ and $\langle p\rangle=0.451$ for self-avoiding unknotted rings [71, 73, 74]. The rings simulated here are not sufficiently long and do not approach such limit values. 